\title{
Los vidrios de baja emisividad
} \author{
MANUEL OLIVARES SANTIAGO, Profesor Titular
Dept. Construcciones Arquitectónicas I, de la Universidad de Sevilla. \\ RAFAEL OLMO ESCRIBANO, Arquitecto \\ ESPAÑA
}

\section{RESUMEN}

Aunque en Europa y EEUU el uso de los vidrios de baja emisividad no es reciente (se llevan utilizando unos 10-15 años), en España son prácticamente desconocidos desde el punto de vista teórico y práctico, a pesar de estar comercializándose desde hace varios años por las casas especializadas.

Nos encontramos con un vacio normativo sobre las condiciones que han de cumplir, ya que la norma de acondicionamiento térmico de los edificios (CT-79) hace referencia a propiedades genéricas de los huecos acristalados, pero no está suficientemente aproximada al comportamiento real de los huecos acristalados con vidrios de baja emisividad y otros elementos semitransparentes.

No es frecuente todavia considerar el comportamiento energético y las características luminosas de los huecos. En los vidrios de baja emisividad estas propiedades ópticas sufren una variación con respecto al vidrio base o soporte debido a la capa de baja emisividad.

Este articulo está estructurado en tres partes. En la primera se analizan las características funcionales y factores ópticos de los vidrios en general.

En la segunda se explica qué son los vidrios de baja emisividad, y cómo se obtienen (esto de forma somera), ya que según el proceso de obtención de la capa de baja emisividad queda limitada en mayor o menor grado la trabajabilidad de los vidrios de baja emisividad.

Finalmente la tercera parte está dedicada al comportamiento térmico de los acristalamientos en general, y en particular a los huecos acristalados, considerándose el vidrio y la carpintería como una unidad funcional.

\section{SUMMARY}

The use of low-emittance coating (low-E) glasses in Europe and the United States is not recent (they make use of them long since: 10-15 years), nevertheless they are almost unknown in Spain, in all respects, in spite of being commercialized long since by differents houses, specialists in these matters.

We run into a lack of regulation about the qualities and stipulations these elements must comply with, since the regulations governing the thermal insulation of the buildings (CT-79) refers to the generic and peculiar qualities of the glazed openings, but these regulations do not approach the question of the real behaviour of the glazed openings with low-emittance glasses and other semi-transparents elements.

The expenditure of energy and the lighting characteristics of the openings are not yet unforeseen, so far. These properties under go a variation with regard to the baseglass or the support, due to the low-emittance coating.

The article is organized in to three parts. The first part is the analysis of the functional characteristics and the lighting properties of the glasses from a general point of view.

The second one explains what low-emittance glasses are, and the way of obtaining them (very briefly); according to the kind of process of obtaining the low-emission coating, the proceedings of low-emission glasses are gradually limited.

Finally, the third part deals with the thermic characteristics of whole glazing, and specially with the glazed openings, from the point of view of a functional consistency for both glass and joinery. 


\section{P A R T E I . - P A R A M E T R O S CARACTERÍSTICOS}

Si nos interesamos por las propiedades de los cerramientos de los edificios (incluidas cubiertas), constatamos que sus propiedades energéticas y luminosas dependen esencialmente de uno de sus elementos, que llamamos acristalamiento, y más concretamente del material tradicionalmente utilizado para realizarlo: el vidrio.

Son los factores ópticos del material los que proporcionan al acristalamiento sus características funcionales en el dominio térmico y luminoso, por ello mostraremos los diferentes factores ópticos, desarrollando su identificación y forma de medida, para ilustrar a la vez la importancia que revisten y la dificultad para su correcto conocimiento.

Por fin, las conclusiones darán luz a las lagunas que hay que rellenar para que la unión "factores ópticoscaracterísticasfuncionales" estébien asegurada para todos los tipos de acristalamientos en innovación.

\section{CARACTERÍSTICAS FUNCIONALES Y} FACTORES ÓPTICAS.

\section{1.- Comportamiento del material frente a la radiación}

Un material transparente cualquiera, recibiendo una radiación de longitud de onda $\lambda_{i}$, se puede comportar de tres maneras diferentes (Fig. 1):

- Puede reflejar la radiación, en donde la longitud de onda de la radiación no será generalmente modificada.

- Puede transmitir la radiación, en donde la longitud de onda será también la misma. En estos dos casos el material quedará a la misma temperatura.

- Y puede absorber la radiación. Si absorbe la radiación, su temperatura aumentará y se pondrá superior que la de su entorno; se producirá entonces una emisión de la radiación cuya longitud de onda dependerá de la temperatura que alcance el material.

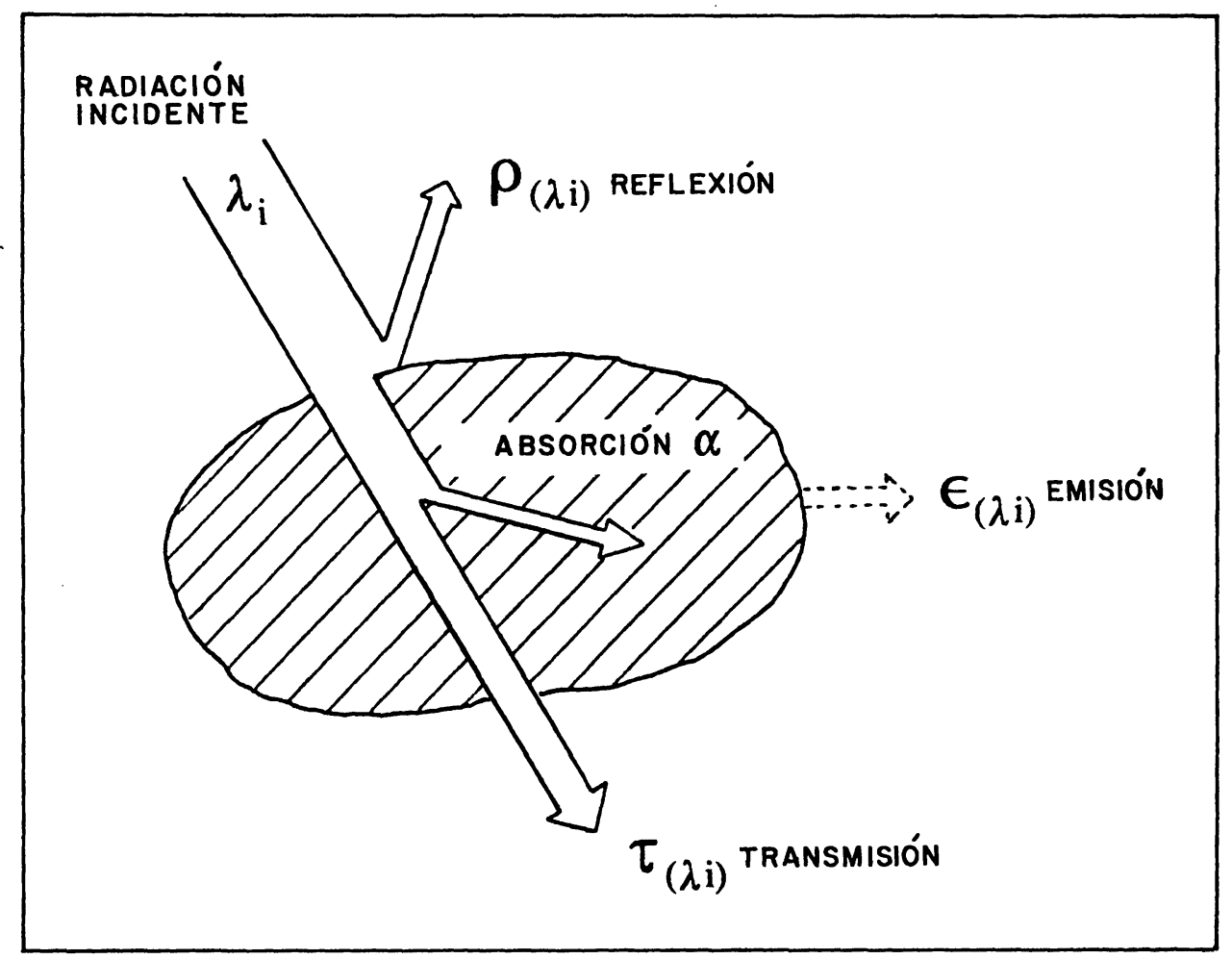

Fig. 1.-Comportamiento de un material cualquiera frente a la radiación. 
En los casos generales, estos fenómenos intervienen simultaneamente y las cantidades de radiación reflejada, transmitida, absorbida y reemitida serán determinadas por unos factores que llamaremos factores ópticos, que son las relaciones de los flujos energéticos transmitidos, reflejados, absorbidos y reemitidos con el flujo energético incidente:

* Factor de reflexión ( $\rho$ ): depende solamente de la superficie que recibe la radiación incidente.

* Factor de transmisión $(\tau)$ : depende de la estructura del material, y del espesor atravesado.

* Factor de absorción $(\alpha)$ : y factor de emisión $(\varepsilon)$ : son propiedades de la superficie del vidrio.

Los factores están unidos por ciertas relaciones de las cuales las principales son:

$$
\begin{gathered}
\alpha+\beta+\tau=1 \\
\alpha=\varepsilon
\end{gathered}
$$

para una misma longitud de onda, pero estas relaciones no son verdaderas en todo su rigor, ya que solamente son ciertas cuando los factores ópticos están completamente identificados.

\section{2.- Identificación completa de un factor óptico}

Un factor óptico está completamente identificado cuando se ha definido a la vez su geometría y su dominio espectral. La información sobre la gama de longitud de onda (o dominio espectral) sobre la que opera el factor óptico se expresa por un subíndice. Estos subíndices son:

-" $\lambda$ " quiere indicar espectral, es decir, para un longitud de onda dada.

-"v"(o "l") significa que el factor está integrado sobre el dominio espectral visible (o luminoso): entre 0,38 y $0,78 \mu \mathrm{m}$.

-"s" corresponde a una integración sobre el dominio solar $(0,35$ y $2,1 \mu \mathrm{m})$.

-"th" designa de manera general el dominio térmico (infrarrojo) entre 5 y $50 \mu \mathrm{m}$. Si queremos precisar, podemos dar un nombre (por ejemplo “100"): en este caso el factor está integrado en el dominio espectral correspondiente al de la emisión de un cuerpo negro elevado a $100^{\circ} \mathrm{C}$ de temperatura.

Lo referente a la geometría de radiación se expresa por dos superíndices, es decir, que para la reflexión y la transmisión hay dos superíndices. El primero es para la radiación incidente y el segundo para la radiación reflejada.

El superíndice del factor de absorción concierne a la radiación incidente, y el del factor de emisión concierne a la radiación emitida:

-"n" quiere decir normal a la superficie del material.

-"h" quiere decir hemisférico (proveniente del todo el hemisferio o detectado sobre todo el hemisferio encima del material)

-"d" quiere decir direccional: también se usa el símbolo "prima" (").

Algunos factores son particularmente interesantes:

-El factor de reflexión especular por ejemplo designa el factor de reflexión direccional-direccional en la que la dirección reflejada es simétrica de la dirección de la radiación incidente con relación a la superficie.

-El factor de transmisión direccional-direccional en la dirección de la radiación incidente se llama factor de transmisión regular.

En estos dos últimos casos, se ha tenido en cuenta la radiación directa. Si queremos considerar la radiación difusa (por transmisión o por reflexión) se necesita una detección hemisférica.

La figura 2 ilustra algunos ejemplos de factores ópticos utilizados, para los cuales se admite generalmente una notación abreviada.

La figura 3 da los valores de factores usuales en el caso de cristal ordinario.

\section{3.- Características funcionales de un acristalamiento}

Después de haber abordado con generalidad los factores ópticos de los materiales transparentes pasemos a considerar el punto de vista opuesto, es decir, el del observador colocado detrás de una superficie acristalada. 
FUENTE $(0,35 \div 2,1 \mu \mathrm{m})$

FUENTE $(0,35 \div 2,1 \mu \mathrm{m})$

FACTOR DE ABSORCIÓN SOLAR NORMAL: $\boldsymbol{a}_{s}^{n}$

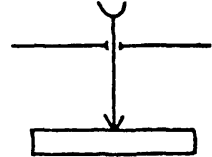

NOTACIÓN ABREVIADA

FACTOR DE ABSORCIÓN SOLAR $\boldsymbol{\alpha}_{S}$

$$
\text { FUENTE }(0,35 \div 2,1 \mu \mathrm{m})
$$

FACTOR DE REFLEXIÓN SOLAR BIDIRECCIONAL: $\rho_{S}^{d d}$

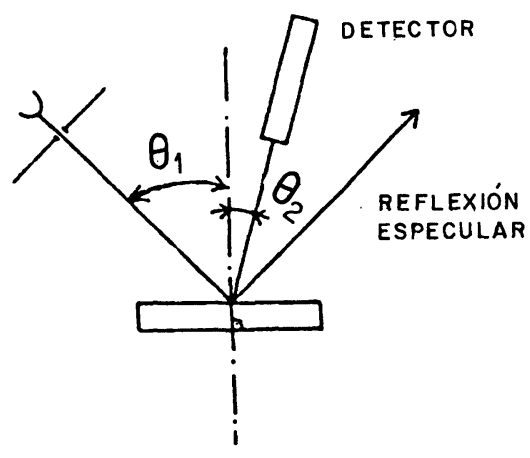

FACTOR DE TRANSMISIÓN SOLAR NORMAL HEMIESFÉRICO: $\tau_{s}^{\text {hn }}$

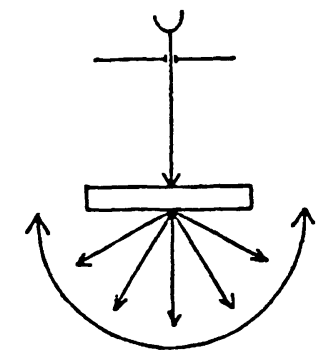

NOTACIÓN ABREVIADA:

FACTOR DE TRANSMISIÓN SOLAR $\tau_{S}$

FACTOR DE EMISIÓN TÉRMICA HEMIESFÉRICO: $\boldsymbol{\epsilon}_{\text {th }}^{h}$

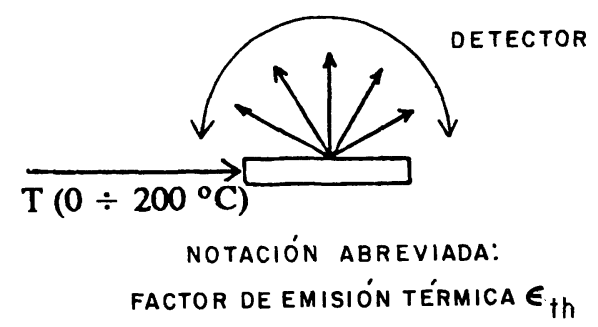

Fig.2.- Algunos factores ópticos usuales y sus notaciones abreviadas.

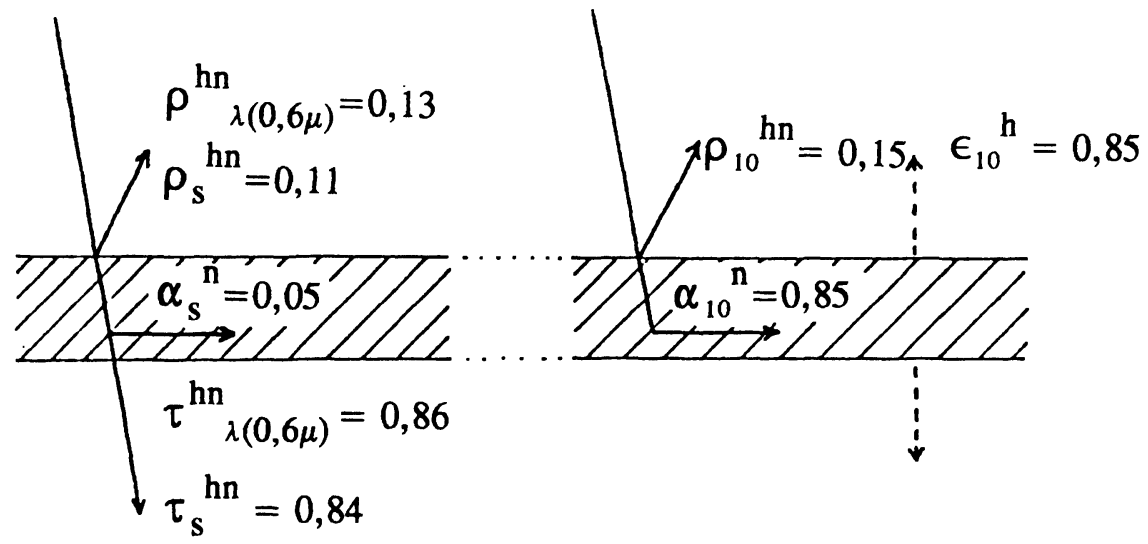

DOMINIO SOLAR

DOMINIO INFRARROJO

Fig.3.- Los valores de los factores ópticos del cristal en los dominios solar e infrarrojo (valores medios para un cristal de $4 \mathrm{~mm}$ de espesor). 
Las funciones aseguradas por una superficie acristalada en un edificio son múltiples. Si sólo se tienen en cuenta los aspectos térmicos y luminosos, la figura 4 ilustra lo que llamaremos las 3 características funcionales de un acristalamiento. Se trata de la transmisión luminosa $\left(\tau_{\mathrm{v}}\right)$, del factor solar (S) y de la transmisión térmica superficial (K).

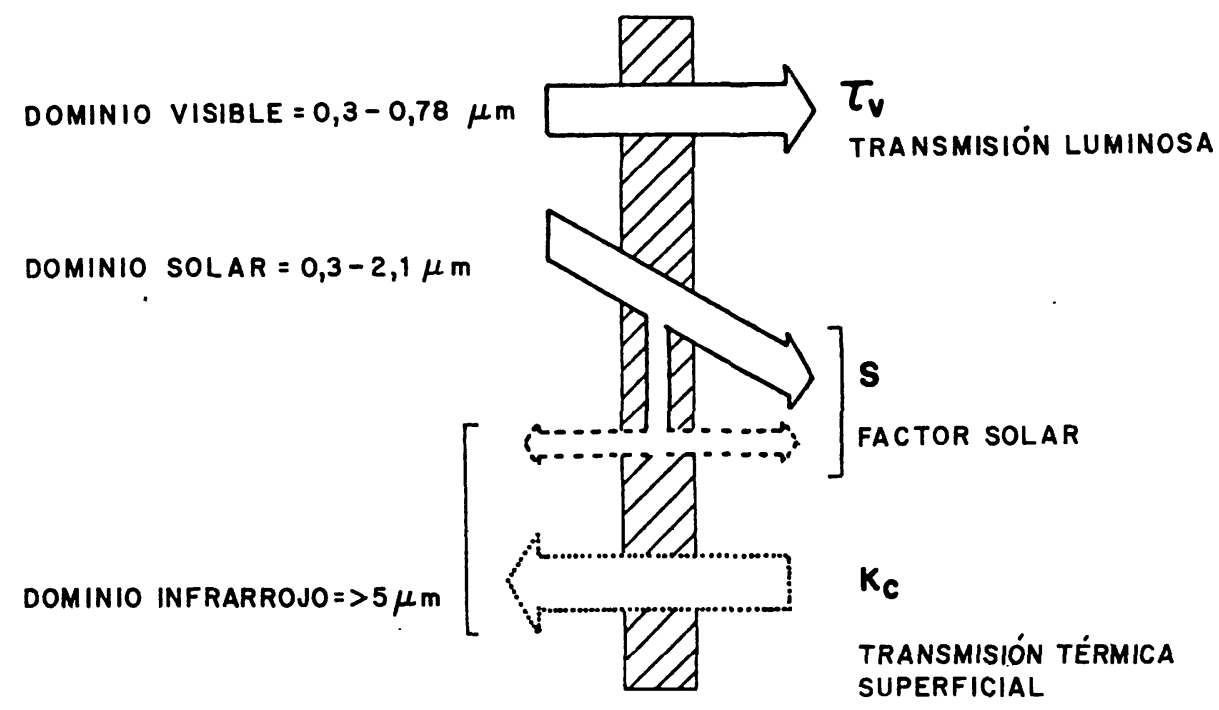

EXTERIOR

INTERIOR

Fig.4.- Las características funcionales de un acristalamiento.

La eficacia luminosa de las diferentes radiaciones permite transformar el flujo energético emitido por una fuente de rayos en flujo luminoso. Para la luz solar es suficiente multiplicar la cantidad de energía presente en cada radiación monocromática, por su eficacia luminosa $\mathrm{W}$, siendo $\mathrm{W}$ el factor de ponderación que tienen en cuenta el "luminoso de referencia CIE D65" (cercano al sol) y el "observador de referencia CIE", que ha sido normalizada por la "COMISIÓN INTERNACIONAL DE LA ILUMINACIÓN”. El dominio espectral es el de la luz visible.

De la energía transportada por una onda luminosa al incidir sobre un vidrio en cualquier dirección, una parte es reflejada, otra es absorbida en su masa, y el resto, emerge en la misma dirección después de sufrir una refracción.

Los factores de transmisión y de reflexión luminosa de un vidrio son las relaciones del flujo luminoso transmitido o del flujo luminoso reflejado, al flujo luminoso incidente

La transmisión luminosa " $\tau_{\mathbf{v}}$ " es la fracción de luz natural que atraviesa el acristalamiento. Interviene en el cálculo de las necesidades de iluminación artificial del edificio.

El factor solar "S" es la relación entre la energia total que entra en un local a través de un acristalamiento y la energía solar que incide sobre dicho acristalamiento. El factor solar (suma de la transmisión solar directa «energía solar que entra por transmisión directa» y de la fracción de energía absorbida por convección y radiación «cedida por el acristalamiento al interior del local como consecuencia de la absorción del cristal») es determinante sobre las cargas de climatización:

-si se reduce, permite en verano evitar los sobrecalentamientos indeseados,

-por el contrario si tenemos un factor solar elevado nos permite en invierno beneficiarnos de los aportes térmicos gratuitos.

Las recomendaciones españolas del CITAV parael cálculo del factor solar consideran:

-El sol en plano vertical normal a la fachada, a una altura de $30^{\circ}$ por encima del horizonte. Es a lo que el proyecto de norma francesa a la que se hace referencia el apartado siguiente llama "factor solar útil".

-La temperatura ambiente interior y exterior iguales entre ellas.

-Los coeficientes de cambio del vidrio hacia el exterior, $h_{e}$ 
$=20 \mathrm{kcal} / \mathrm{h} \mathrm{m}^{2}{ }^{\circ} \mathrm{C}$ y hacia el interior, $\mathrm{h}_{\mathrm{i}}=7 \mathrm{kcal} / \mathrm{h} \mathrm{m}^{2}{ }^{\circ} \mathrm{C}$

Por últimoel coeficiente de transmisión térmica superficial " $K_{c "}$ (la cantidad de calor transmitida por una unidad de superficie durante una unidad de tiempo en régimen estacionario para una diferencia de temperatura de $1^{\circ} \mathrm{C}$ entre sus caras) es la característica más tradicional y permite el cálculo de pérdidas térmicas de un edificio.

\section{4.- Caso de un doble acristalamiento}

El conocimiento de las características evocadas en el

$$
\mathcal{T}_{v}=\frac{\int_{0.38}^{0.78} \overline{\mathcal{I}_{\lambda}} W_{\lambda} d_{\lambda}}{\int_{0.38}^{0.78} w_{\lambda} d_{\lambda}}
$$

donde

$$
K_{c}, h_{e}, h_{c} \text { son funciones de } \varepsilon_{1}, \varepsilon_{2}, \varepsilon_{3}
$$

con

$$
\begin{gathered}
\mathcal{T}_{\lambda}=\frac{\left.\mathcal{T}_{1 \lambda}\right]}{1-\rho_{1 \lambda} \rho_{2 \lambda}} \\
S=\mathcal{T}_{e}+q_{i} \\
q_{i}=\alpha_{e} \frac{K_{c}}{h_{e}}+\alpha_{i} \frac{K_{c}}{h_{c}} \\
\mathcal{T}_{e}=\frac{\int_{0.35}^{2.1} \mathcal{T}_{\lambda} S_{\lambda} d_{\lambda}}{\int_{0.35}^{2.1} S_{\lambda} d_{\lambda}}
\end{gathered}
$$

apartado anterior es esencial para el uso que se hará de la superficie acristalada. Se obtienen mediante un cálculo en el que se introducen los diferentes factores ópticos. Se dan en la figura 5 las diferentes fórmulas (simplificadas) que permiten realizar esos cálculos.

El cálculo detallado puede obtenerse en los siguientes textos:

*Para el coef. $\mathrm{K}_{\mathrm{c}}$ : "Règle de calcul des caractéristiques thermiques utiles des parois de construction" (Reglas de cálculo de características térmicas útiles de paredes de$$
\frac{1}{K_{c}}=\frac{1}{h_{i}}+\frac{1}{h_{e}}+\sum \frac{e}{\lambda}+\sum R \quad\left(m^{2} \frac{C}{W}\right)
$$$$
\frac{1}{R}=h_{c}+h_{r} \quad h_{r}=\frac{5.14}{1} \frac{1}{\varepsilon_{2}+\varepsilon_{3}-1}
$$$$
\operatorname{con}
$$$$
h_{c}, h_{i} \text { función de } \varepsilon_{1}, \varepsilon_{4}
$$

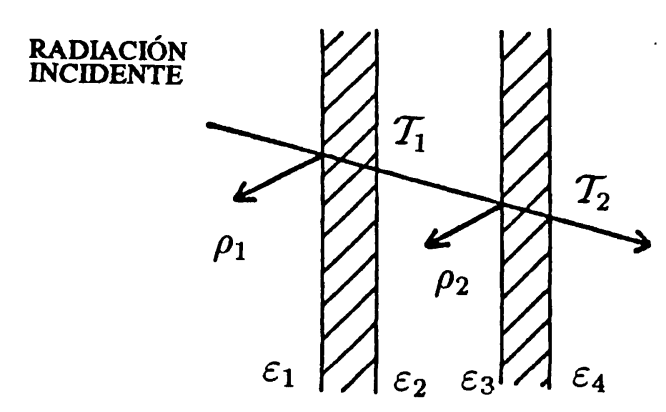

EXTERIOR

INTERIOR

Fig.5.- Los factores ópticos (encuadrados) en los cálculos de las características funcionales (caso de un doble acristalamiento). 
construcción), Libreta del CSTB n ${ }^{\circ} 2096$ de septiembre de 1986.

* Para el factor solar y la transmisión luminosa: "Détermination du facteur de transmission lumineuse et du facteur solaire conventionnel des vitrages" (Determinación del factor de transmisión luminosa y del factor solar convencional de los acristalamientos", Proyecto de norma francesa de Junio de 1987 . No hay norma UNE a tal efecto.

La figura 5 tiene por único fin el mostrar dentro de los cálculos, el sitio de los diferentes factores ópticos, los cuales aparecen recuadrados, y la importancia de su identificación. Trata el caso clásico de un doble acristalamiento.

Para la transmisión luminosa hay que introducir en el cálculo los factores de transmisión y de reflexión de cada cristal. El proyecto de norma francesa no da las características geométricas de los factores considerados; parecería lógico retener los factores normales hemisféricos aunque en la práctica se utilizan más bien los factores normales-normales.

Para el factor solar el factor $\tau_{\lambda}$ debe ser esta vez integrado sobre la totalidad del espectro solar: se ve en la figura 3 que para el cristal es ligeramente diferente del factor integrado solamente en el dominio visible. Ya que ninguna recomendación aparece en el documento de referencia, sería bueno retener aquí también los factores normales hemisféricos.

Un acercamiento más fino al factor solar lo podríamos llamar "factor solar útil". Consideraría los factores solares direccionales de $30^{\circ}$ de incidencia para estar más cerca de las condiciones medias de soleamiento de una superficie acristalada.

En el cálculo del $\mathrm{K}_{\mathrm{c}}$ debemos introducir los factores de emisión de cada cara. El documento precisa que conviene dar los factores hemisféricos. El dominio de longitud de onda no precisado es el de la emisión de un cuerpo negro a temperatura ambiente, para acercarse a las condiciones de empleo de un acristalamiento.

La finalidad de esta exposición no es la de insistir sobre los métodos de cálculo, sino sobre la importancia de identificación de los factores ópticos pertinentes: si en el caso de un vidrio ordinario podemos introducir los valores estándar para los diferentes factores, convendría para otros materiales y sobre todo para acristalamientos novedosos, determinar con precisión los factores ópticos.
Esta determinación se hace en dos etapas:

-La identificación: acabamos de ver que es importante precisar las características espectrales y geométricas de cada factor.

-La medida: será necesario para los materiales nuevos. Vamos a ver con un ejemplo que da algunos problemas.

\section{LOS ACRISTALAMIENTOS CON CAPAS POCO EMISIVAS.}

Estos acristalamientos toman una parte creciente del mercado del doble acristalamiento. Volvamos alas fórmulas de la figura 5 , considerando sus propiedades específicas.

\section{1.- Transmisión y reflexión}

Para la transmisión luminosa hay que medir los factores de transmisión y de reflexión en el dominio visible. $\mathrm{Si}$ queremos hacer la hipótesis de que ninguna luz es difusa por la capa de baja emisividad, la medida será de factores normales-normales. Si existe duda, la detección debe ser hemisférica: debemos medir entonces los factores normales hemisféricos.

Para el factor solar el dominio espectral de medida de factores de transmisión y de reflexión se extiende hasta $2.500 \mathrm{~nm}$, pero en lo que concierne a la geometría se aplican las conclusiones anteriores. Si nos interesamos por los factores direccionales para una incidencia de $70^{\circ}$ podemos aceptar para el acristalamiento con capas las correlaciones conocidas para el vidrio, y podemos calcular ese nuevo factor. Pero pensamos que ciertas capas tienen alguna incidencia diferente de la normal, un comportamiento específico, y será entonces útil tomar la medida real a incidencia variable; existe no obstante un problema de instrumentación, porque ningún instrumento del mercado permite medir el factor de transmisión direccional hemisférico a diferentes incidencias.

Las curvas de las Figs. 6 y 7, ilustran lo que se acaba de decir. Se trata de los factores espectrales de transmisión y de reflexión para tres materiales: un cristal ordinario, un cristal con capa bajo emisiva tipo pirolítica y un cristal con capa bajo emisiva tipo metálico o catódica.

El aparato utilizadoes un espectofotómetro BECKMANN 5240 UV-VIS-NIR equipado de una esfera integrante de $150 \mathrm{~mm}$ de diámetro revestida de una capa difusora de $\mathrm{BaSO}_{4}$.

Las fórmulas de las curvas, así como los valores integrados 
para losfactores en los dominios visibles y solar de la tabla siguiente, muestran que si la confusión entre el factor solar y el factor luminoso se puede aceptar para el vidrio ordinario, no lo es del todo para los cristales con capas.

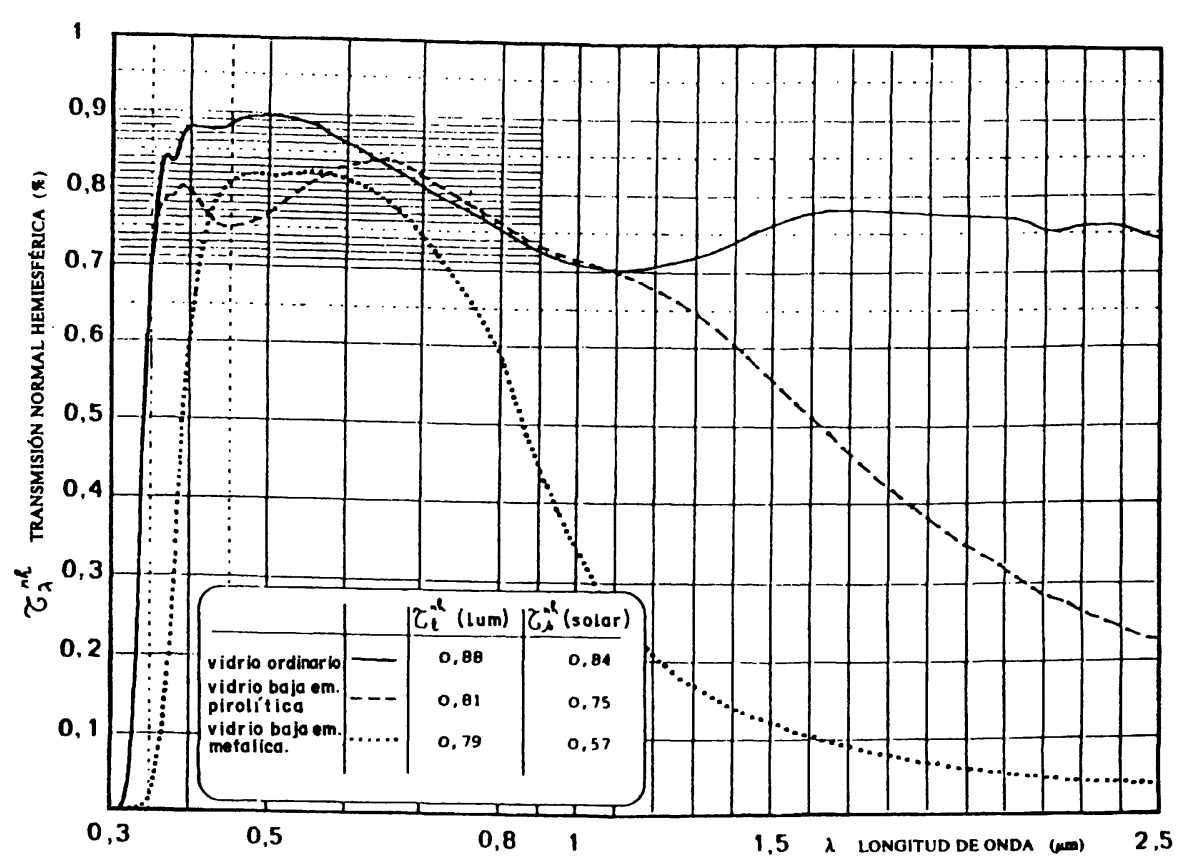

Fig.6.- Curvas de transmisión normal hemiesférica para tres tipos de vidrios.

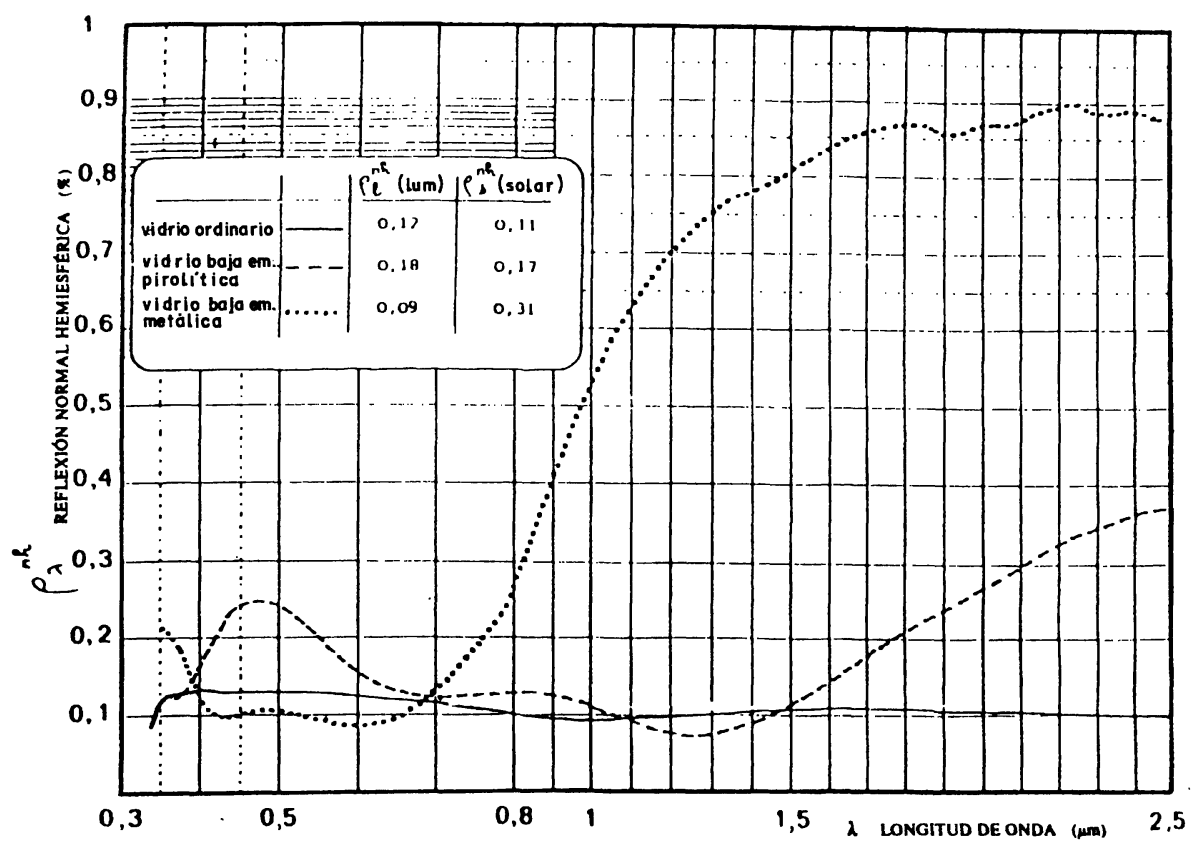

Fig.7.- Curvas de reflexión normal hemiesférica para tres tipos de vidrios.

\begin{tabular}{|l|c|c|c|c|}
\hline \multirow{2}{*}{} & \multicolumn{2}{|c|}{ Factores de transmisión } & \multicolumn{2}{c|}{ Factores de reflexión } \\
\cline { 2 - 5 } & Luminoso & Solar & Luminoso & Solar \\
\hline Cristal ordinario & 0,88 & 0,84 & 0,12 & 0,11 \\
\hline C. con capa pirolítica & 0,81 & 0,75 & 0,18 & 0,17 \\
\hline C. con capa metálica & 0,79 & 0,57 & 0,09 & 0,31 \\
\hline
\end{tabular}




\section{2.- Factor de emisión térmica}

Para el cálculo del coef. $K_{c}$ hay que disponer del factor de emisión hemisférico, cuya medida es delicada en ausencia de norma de mediday de instrumentación verdaderamente adaptada. Entre las técnicas presentadas en la figura 8, la mayoría permiten acceder al factor de emisión térmica por la medida del factor de reflexión infrarroja, que es la adoptada por los cristaleros y que entra en un proyecto de norma ISO; esta forma de medida se interesa por la reflexión especular, lo que equivale a hacer la hipótesis de que las superficies consideradas no son difusoras. Nos podemos interrogar sobre la validez de esta hipótesis para ciertas capas.

Los resultados obtenidos utilizando cada uno de estos métodos presentan una dispersión que no es aceptable: los valores del factor de emisión se dispersan de 0,30 a 0,45
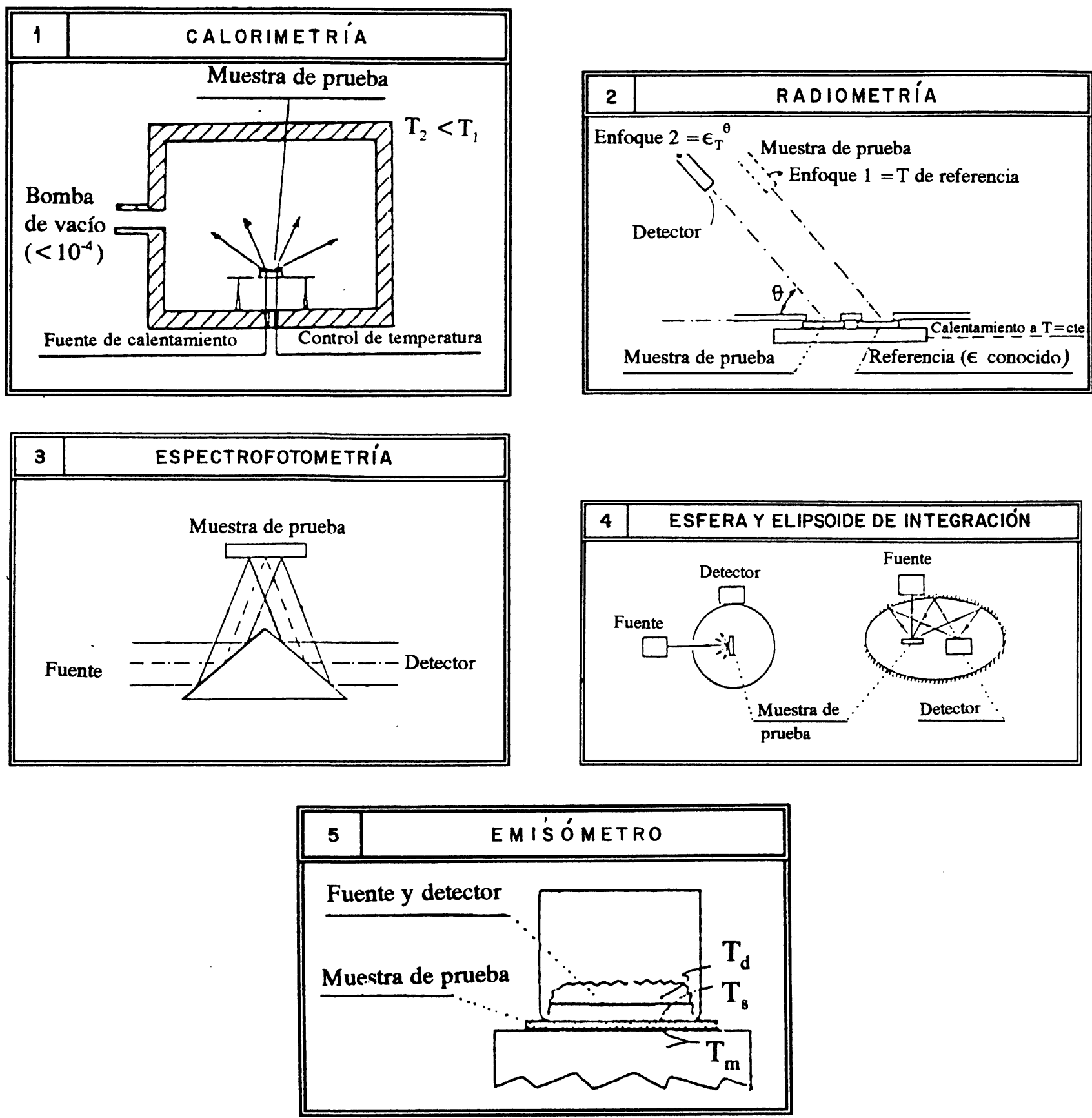

Fig.8.- Técnicas de medida del factor de emisión térmica. 
para una misma capa pirolítica y de 0,06 a 0,17 para una misma capa metálica.

El único método que permite una medida directa del factor de emisividad es el método calorimétrico.

No sería razonable proceder a una medida calorimétrica para cada determinación, a causa de la complejidad de este método, pero parece necesario disponer de un emisómetro de tipo calorimétrico adaptado a una medida directa a la temperatura ambiente; tal aparato permitiría medidas de referencia y la caracterización de un modelo para los aparatos más simples, lo que mejoraría su fiabilidad: tal aparato adaptado a materiales como el cristal no existe actualmente.

\section{ALGUNOSMATERIALES SEMITRANSPARENTES O TRANSLÚCIDOS.}

Los problemas puestos en evidencia en el apartado precedente en lo que concierne a los acristalamientos con capa bajo emisiva, que resultan a la vez de la dificultad de identificar el factor óptico pertinente y de encontrar una instrumentación adaptada, son aún más acentuados cuando abordamos otros tipos de materiales que aparecen ahora en los dominios del acristalamiento en un sentido muy amplio. Hablemos rápidamente de películas de plásticos multicapa y de aislantes llamados transparentes.

\section{1.- Las películas}

Diversidad de productos se presentan en el mercado para llenar varias funciones: antirreflejo, protección solar, baja emisividad,... . Conviene verificar estas diferentes propiedades, que no se pueden reunir en un solo producto. La medida de la transmisión y de la reflexión al espectrofotómetro se perturba por los fenómenos de reflexión múltiple y de interferencias para las películas muy delgadas (algunas decenas de micras), y la utilización de curvas para el cálculo de los factores integrados (solar o luminoso) se debe de hacer con precaución. La figura 9 muestra un ejemplo del resultado sobre una película actualmente comercializada.

Para el factor de emisión las precauciones son igualmente necesarias, sobretodo con los emisómetros portátiles: la medida sobre la película sola puede ser falsa porque la transmisión infrarroja no es siempre nula por el pequeño espesor, y la medida sobre soporte de cristal supone una aplicación correcta (difícil sobre pequeñas superficies). La medida es aun más perturbada por la conductividad térmica del cristal.

\section{2.- Los plásticos multicapas}

Distinguimos por este término los materiales constituidos

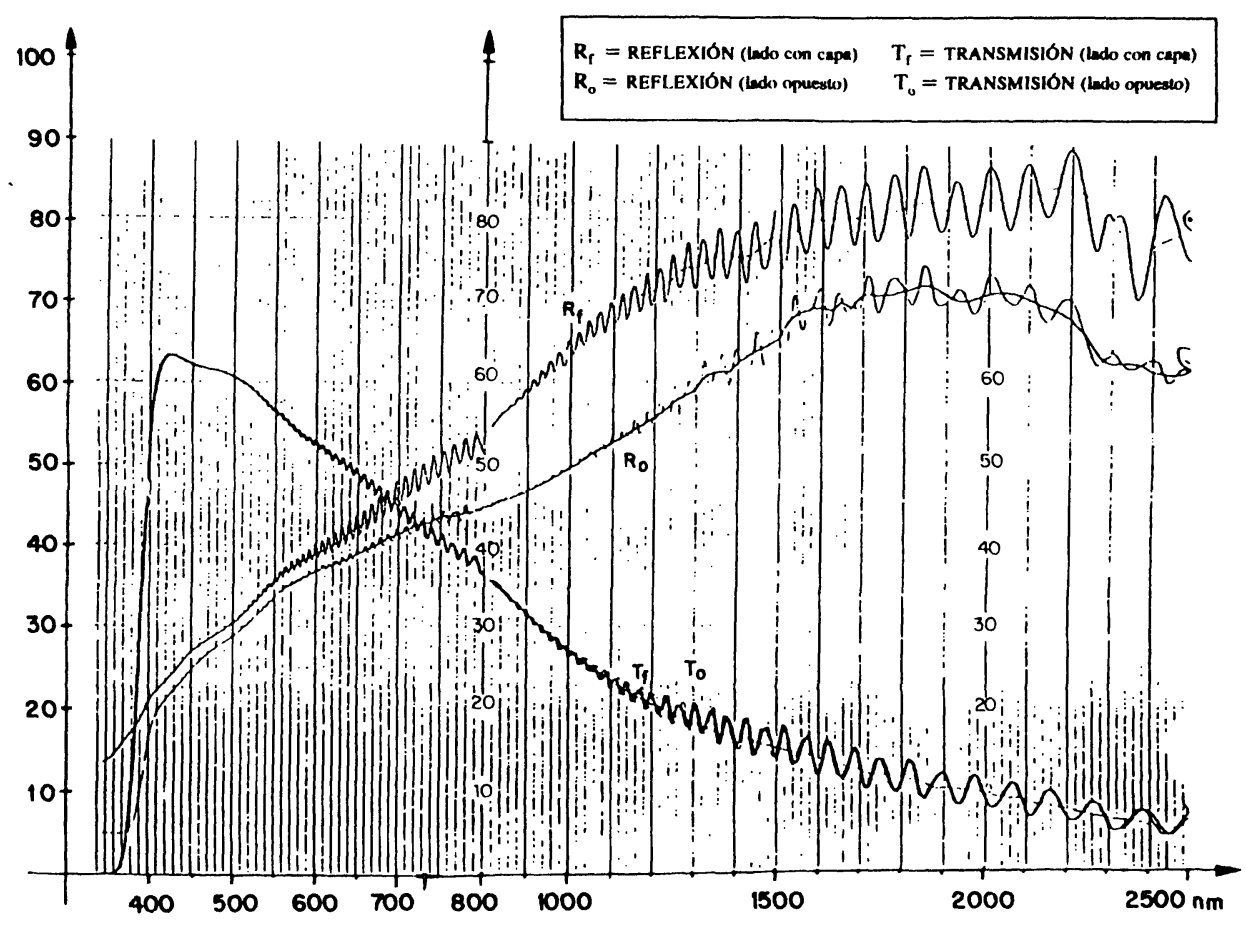

Fig.9.- Curvas de transmisión y de reflexión normal hemiesférica de una capa depositada sobre vidrio. 
por hojas delgadas de materia plástica transparente (PMMA, policarbonato) unidas entre ellas por nervios para darle una rigidez suficiente. Estos materiales permiten en ciertos locales la entrada de luz natural sin los inconvenientes del cristal. Para estos materiales la medida de la transmisión luminosa tiene dos conclusiones:

-La medida del factor normal-normal (es decir, sin esfera de integración) puede ser totalmente falsa, siguiendo el punto de impacto del haz. Como ya se ha explicado los textos noexigen una detección hemisférica para caracterizar este tipo de material.

-El factor de transmisiónen función del ángulode incidencia es para estos materiales una característica muy interesante, pero esta medida como hemos dicho anteriormente no es posible con los aparatos clásicos.

\subsection{Los aislantes transparentes}

Todavía en estado de prototipo estos materiales (Gel de sílice, multicapa de 7 u 8 hojas, espuma de PMMA, ...) aunan una transmisión luminosa importante a una débil conductividad térmica. En lo que concierne a sus propiedades ópticas la dificultad es doble:

-su heterogeneidad macroscópica exige una superficie de medida (una muestra de prueba) fuera de proporción con los aparatos clásicos;

-el grosor de estos productos (como mínimo $10 \mathrm{~mm}$ ) es una causa suplementaria de error, ya que los bordes juegan un papel importante, porque las reflexiones en todas direcciones son significativas.

Para estos materiales la redacción de una norma de medida es aún prematura, pero la construcción de un aparato específico con esfera de integración de gran diámetro es una urgencia.

\section{CONCLUSIONES.}

Después de haber presentadolos diferentesfactores ópticos y descrito su complejidad, los hemos situado en los cálculos de características funcionales de una superficie acristalada. También hemos insistido sobre la evolución reciente de este dominio. La penetración en el mundo del edificio de acristalamientos llamados innovadores es ahora significativa, y debería acentuarse aún más. Aquí los arquitectos y los diseñadores teníamos a nuestra disposición un único material: el cristal, y ahora podemos escoger entre un número creciente de materiales, incluso si el cristal queda como base.
Pero lo que diferencian estos materiales nuevos son justamente las propiedades ópticas. Las costumbres deben igualmente evolucionar para el cálculo de características funcionales de los mismos y no nos debemos de contentar con utilizar valores estándar de factores ópticos; conviene por tanto identificar el factor óptico pertinente y medirlo.

Hemos puesto en evidencia algunas lagunas respecto a este propósito, tanto desde el punto de vista de los textos normativos o reglamentarios, como desde el punto de vista de instrumentación que permita las medidas.

La realización en el CSTB con ayuda de AFME de una plataforma de medida para las propiedades ópticas y radiantes de materiales, llamada OPTORA debe permitir resolver buena parte de los problemas de dispositivos de medida. El trabajo que se realizará sobre esta plataforma permitirá a estos dispositivos ser aplicables a todos los materiales innovadores para acristalamientos.

\section{PARTE II.- LOS VIDRIOS DE BAJA EMISIVIDAD}

¿Qué son los vidrios de baja emisividad?

Son lunas de vidrio ordinario en las que una de sus caras ha sido tratada con precipitaciones de sales, óxidos metálicos y metales nobles, mediante tecnologías avanzadas, para conseguir un coeficiente de emisividad muy inferior al del vidrio base manteniendo una elevada transmisión luminosa. Son vidrios dirigidos a limitar las pérdidas energéticas a través de los acristalamientos, aumentando el aislamiento y facilitando las aportaciones solares, permitiendo el paso de la energía solar (luz y calor) y reflejando el infrarrojo lejano.

Los sistemas de fabricación más populares son:

-Depositación pirolítica en caliente (directamente en el horno de fabricación del vidrio base).

Más exactamente es un depósito por pirólisis en fase sólida. Es un procedimiento y tecnología desarrollada por SAINT GOBAIN para la fabricación de la capa "EKO". Consiste en proyectar sobre el vidrio caliente, mediante un sistema de distribución lineal, un flujo gaseoso portador de granos finamente divididos del compuesto reactivo. Por descomposición pirolítica se forma sobre el vidrio una capa de un semi-conductor transparente, que es la capa de baja emisividad. La ventaja de este depósito es que al incorporarse sobre el vidrio a elevadas temperaturas su adherencia es muy buena (por reacciones entre el propio 
vidrio y los componentes constitutivos de la capa en las superficies límite de contacto). Además de esa importante ventaja, que mejora la resistencia mecánica de las capas, este depósito se realiza en continuo, directamente sobre la línea de fabricación del vidrio, con elevadas cadencias de producción y relativos bajos costes (a velocidades de hasta $20 \mathrm{~m} / \mathrm{min}$ en un ancho de 3,2 metros).

\section{-Pulverización catódica en cámara de vacío.}

El vapor es obtenido por bombardeo de partículas de un gas, parcialmente ionizado, sobre el cátodo del material a depositar, arrancando átomos de éste que se proyectan sobre el substrato. La descarga de los iones positivos del gas produce electrodos secundarios que vuelven a entrar en colisión con los átomos del gas ionizado de nuevo, dando continuidad al ciclo. En el grupo SAINT GOBAIN se sigue un importante programa de investigación para la obtención de multicapas que tengan un buen compromiso entre la transmisión luminosa deseada, un factor solar bajo y la coloración y reflexión exterior elegida. Por este procedimiento se obtiene la familia de capas de baja emisividad tipo "PLANITHERM".

\section{1.- EFECTO INVERNADERO.}

La energía solar que entra en un local a través de un acristalamiento es absorbida por los objetos y paredes interiores, que al calentarse, emiten radiaciones caloríficas de larga longitud de onda (superior a $5 \mu \mathrm{m}$ ).

Los vidrios, al ser prácticamente impermeables a las radiaciones de longitud de onda superior a $5 \mu \mathrm{m}$, hacen que la energía solar que pasa a través de un acristalamiento, se encuentre retenida en el interior del local, tendiendo este a elevar su temperatura. A este fenómeno se le conoce con el nombre de "efecto invernadero". Vease la figura 10.

\section{2.- EMISIVIDAD.}

Los cuerpos al calentarse emiten radiaciones de gran longitud de onda, superior a $5 \mu \mathrm{m}$, a los cuales el vidrio es opaco, es decir, absorbente. Por esta razón técnicamente se les denomina como materiales semitransparentes.

Por las leyes que regulan los cambios térmicos sabemos que la absorción se traduce en un calentamiento, con parte de energía reemitida al exterior y parte al interior, pero la mayor de ella al medio más frío, al exterior en invierno y al interior en verano.

Para disminuir esta pérdida de calor, absorbiday reemitida hacia el exterior en invierno, se han creado los vidrios de baja emisividad que constan en una de sus caras de una

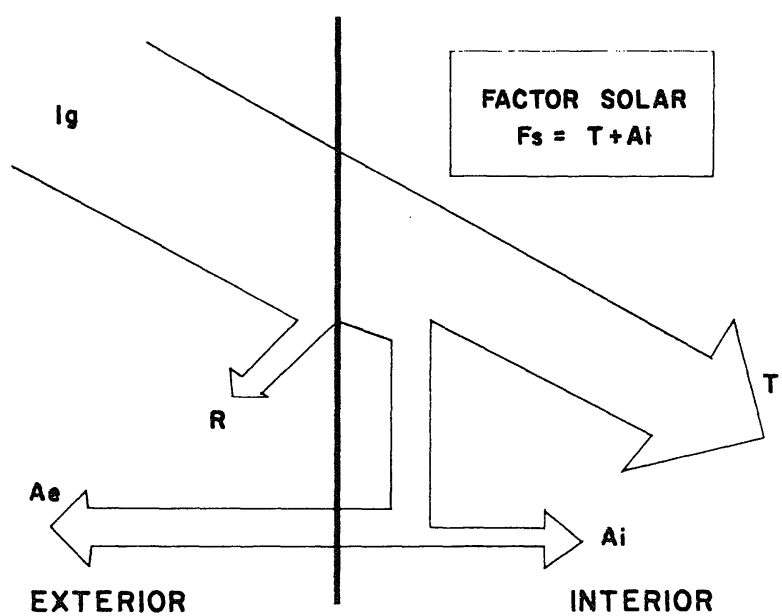

Fig. 10.- Comportamiento de un vidrio ordinario frente a la radiación solar.

capa especial que refleja las radiaciones de gran longitud de onda, superiores a $5 \mu \mathrm{m}$, evitando el paso de las mismas hacia el exterior.

\section{3.- TRANSMISIÓN DE CALOR.}

El intercambio de calor entre el interior de un recinto y el exterior es la suma del intercambio convectivo (entre el aire y la superficie de vidrio, tanto en el interior como en el exterior), y de intercambio radiante (producido por la diferencia de temperatura entre la temperatura del vidrio y la temperatura del medio exterior, ya que la temperatura del recinto y la del aire interior son sensiblemente equivalentes). La expresión que representa el intercambio es:

$q=h\left(T_{v}-T_{a}\right)+4 \sigma T_{m}^{3} \varepsilon\left(T_{v}-T_{r}\right)$

suele darse que $\mathrm{T} \approx \mathrm{T}_{\mathrm{a}}$ con lo que

$q=\left(h+4 \sigma T_{m}^{3} \varepsilon\right)\left(T_{v}-T_{r}\right)$

* $\mathrm{q}$ es el intercambio de calor por $\mathrm{m}^{2}$

* $h$ es el coeficiente de transferencia por convección y normalmente toma el valor $\mathrm{h}=3 / 4$

* $\mathrm{T}_{\mathrm{v}}$ es la temperatura del vidrio

* $\mathrm{T}_{\mathrm{a}}$ es la temperatura del aire interior

* $\mathrm{T}_{\mathrm{r}}$ es la temperatura del recinto

$* 4 \sigma \mathrm{T}_{\mathrm{m}}{ }^{3} \approx 5$ normalmente

$* \varepsilon=0,85$ para vidrio normal

${ }^{*} \varepsilon=0,1 \div 0,35$ en la cara con capa de baja emisividad

Con estos valores genéricos obtenemos que el vidrio normal nos da un valor de intercambio de calor igual a 8,25 y el vidrio con capa de baja emisividad, (considerando una emisividad media) colocada al exterior de 5,25, lo que de 
formaaproximada nos indica que se produce una reducción en el intercambio de calor interior-exterior del $40 \%$ en números redondos. Este ahorro puede incrementarse si el vidrio con capa de baja emisividad se incorpora a un doble acristalamiento, y aún más si el aire de la cámara es sustituido por Argón.

El aislamiento térmico en la edificación queda exigido en la Norma Básica de la Edificación NBE-CT-79 de forma obligatoria, aunque respecto a los huecos acristalados solamente hace referencia a propiedades genéricas, sin estar suficientemente aproximada al comportamiento real de los mismos, y en particular para huecos acristalados con vidrios de baja emisividad y otros elementos semitransparentes. Las características aludidas en la norma son principalmente:

-Coeficiente de transmisión térmica de los cerramientos (en los que están incluidas las puertas y ventanas que pueden ser elementos acristalados).

-Temperatura superficial interior de los cerramientos, de los que se exceptúan los huecos acristalados produciéndose el efecto de "pared fría".

-Conductividad térmica de los materiales, definida por el coeficiente landa $(\lambda)$. La norma fija unos valores estándar genéricos en el anexo II donde no se recogen de forma específica los vidrios de baja emisividad (por ser novedosos); considerando una relación entre la superficie total del hueco y la superficie acristalada independiente del tamaño del hueco. Los valores estándar dados servirían relativamente para huecos pequeños, pero que no son válidos para superficies acristaladas grandes como muros cortinas, siendo necesaria la obtención de la conductividad térmica global de la superficie acristalada específica en estos casos.

-No hace referencia a las condensaciones superficiales en los huecos acristalados, las cuales producen el desecamiento de los locales con la consiguiente pérdida de las condiciones de confort, además de la posible disminución de la iluminación natural y la pérdida de visibilidad interiorexterior en algunos casos, sin despreciar tampoco el

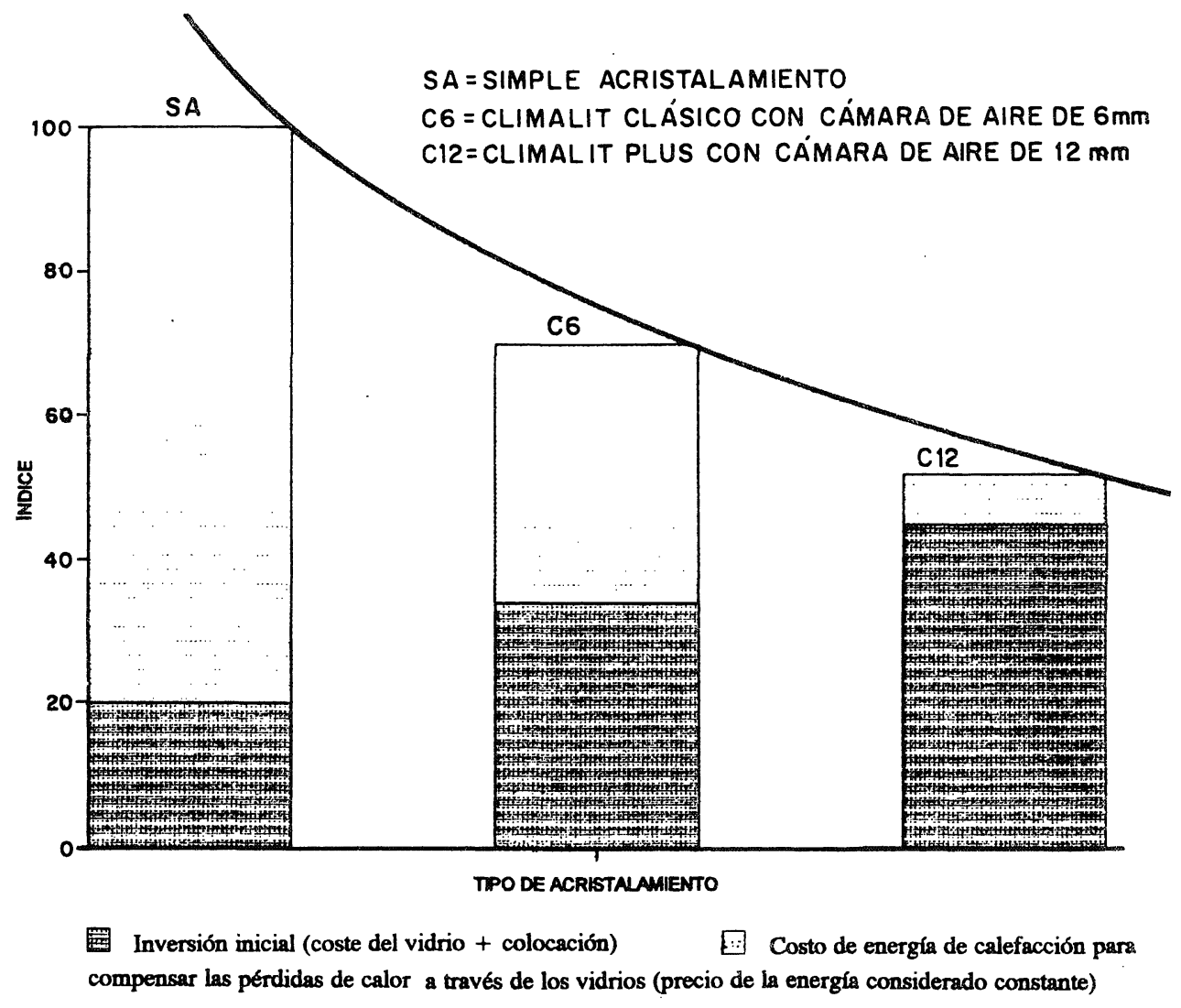

Fig.11.- Balance financiero en pesetas constantes realizado para díez años. 
deterioro del aspecto estético del acristalamiento debido a esa condensación.

Por ser de uso reciente no hay abundante experiencia de sus resultados y se cuenta con referencias de utilización en Estados Unidos y algunas zonas de Europa, donde los diseños de los edificios desde el punto de vista térmico están orientados a las aportaciones o ganancias térmicas, no siendo en general esta premisa válida para España y sobre todo para el sur de la península donde hay una necesidad de aporte energético en invierno y de refrigeración en verano.

Los vidrios de baja emisividad son vidrios que se amortizan en un período pequeño de tiempo, menor cuanto mayor sea la superficie acristalada, por el ahorro energético que producen, a pesar de la mayor inversión inicial, como se recoge en la figura 11, en donde no se ha tenido en cuenta la disminución en el costo de adquisición de los equipos de climatización por la reducción de potencia de los mismos.

El que la capa de baja emisividad impida la reemisión de

ACRISTALAMIENTO SIMPLE

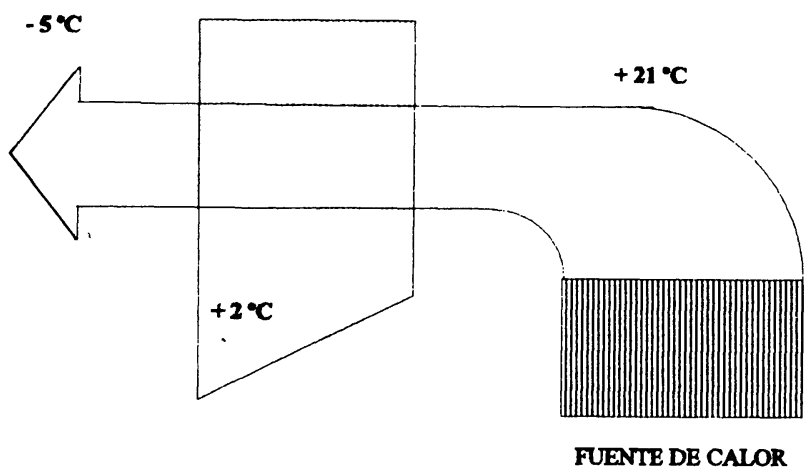

energía absorbida por el vidrio al exterior origina una mejora del coeficiente de transmisión de calor $\mathbf{K}$, todo ello sin detrimento de su Factor Solar y con una pequeña reducción en la transmisión luminosa, lo que permite la captación solar gratuita y el aprovechamiento de la iluminación natural.

\section{4.- ELIMINACIÓN DE CONDENSACIONES}

La mejora del coef. $\mathrm{K}$ produce a su vez el aumento de la temperatura de la cara interna del vidrio, reduciendo el riesgo de aparición de condensaciones y haciendo más confortables las zonas próximas a las superficies acristaladas, eliminando el efecto de "pared fría", como puede apreciarse en la figura 12.

Nos podemos hacer la siguiente pregunta: ¿A qué temperatura exterior aparece el vaho en los cristales de las ventanas?

Esta pregunta se puede responder fácilmente con el diagrama de la figura 13 , siempre que se conozcan las variables siguientes:

\section{DOBLE ACRISTALAMIENTO EMISIVO}

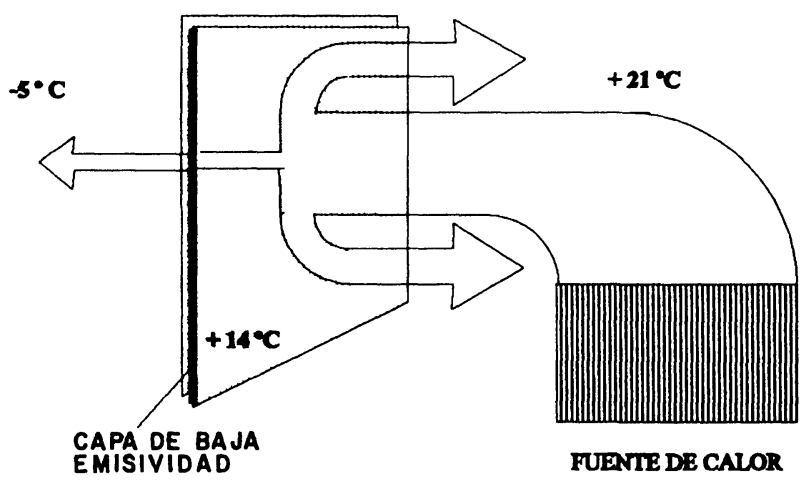

Fig.12.- Temperatura superficial de la cara interna del vidrio. 


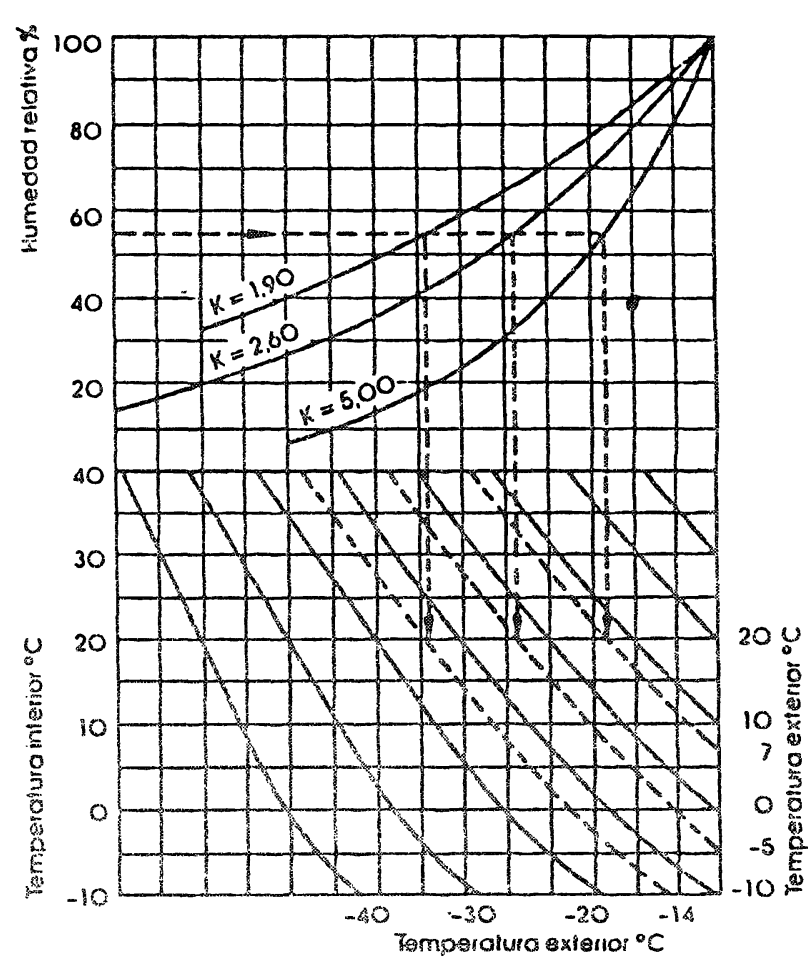

Fig. 13.- Diagrama para comprobación de condensaciones en el vidrio.

- Humedad relativa de aire del local en $\%$

- Temperatura del aire en el local en ${ }^{\circ} \mathrm{C}$

- Valor $\mathrm{K}$ del acristalamiento en $\mathrm{kcal} / \mathrm{h} \mathrm{m}^{2}{ }^{\circ} \mathrm{C}$

Veamos un ejemplo:

Supongamos un local con $\mathrm{HR}=55 \%$ y temperatura del aire en el local $20^{\circ} \mathrm{C}$.- Queremos saber a qué temperatura exterior se empaña un vidrio simple de $\mathrm{K}=5$, y dos dobles acristalamientos con capa de baja emisividad de $\mathrm{K}=2,6$ y $\mathrm{K}=1,9$ (valores corrientes en estos acristalamientos).

Solución.- En el diagrama, partiendo de laHR se traza una líneahorizontal hastacortar la curva del $\mathrm{K}$ correspondiente; desde ese punto se baja una vertical hasta la intersección con la línea horizontal de la $\mathrm{T}^{\mathrm{a}}$. interior y la línea de $\mathrm{T}^{\mathrm{a}}$. exterior que pase por ese punto corresponderá a la $\mathrm{T}^{\mathrm{a}}$. exterior en que comienza a producirse la condensación. Parael acristalamiento simple se producen condensaciones a los $7^{\circ} \mathrm{C}$, y a los $-5^{\circ} \mathrm{C} \mathrm{y}-14{ }^{\circ} \mathrm{C}$ de temperatura exterior para los de baja emisividad respectivamente en el caso de ejemplo considerado.

\section{5.- CONDICIONES DE TRANSFORMACIÓN Y RECOMENDACIONES DE UTILIZACIÓN}

Cuando la capa de baja emisividad es obtenida por precipitación en caliente, la capa resulta una parte integrante de la masa del vidrio, haciéndola inalterable en el tiempo, con resistencia mecánica y estabilidad química, lo que permitiría utilizarlo como un vidrio simple, además de en dobles acristalamientos.

Tienen gran durabilidad y es fácilmente manipulable; no necesita cuidados especiales de embalaje, ni en el transporte, ni en la manipulación, ni en la puesta en obra. Puede sufrir todos los trabajos usuales de transformación como son: templado, curvado, laminado y ensamblado en bloques de doble acristalamiento.

La utilización principal es para edificios residenciales con necesidad de limitar las pérdidas térmicas (y en especial para Arquitectura Bioclimática). Es prácticoy económico. La posibilidad de utilización como vidrio simple sería muy deseable para rehabilitación de edifícios históricos, donde la relación hueco/macizo de los cerramientos es muy pequeño, lo que permitiría la conservación/reutilización de las carpinterías de madera originales de los edificios. Aunque la intención podría ser buena, esta opción no está considerada por las casas comerciales en España, las cuales incluyen siempre los vidrios de baja emisividad en bloques de doble acristalamiento.

Por otro lado, cuando la capa de baja emisividad se obtiene mediante pulverización catódica requiere una manipulación cuidada que garantice la inalterabilidad de la cara tratada. Por ello solamente es recomendabie en unidades prefabricadas de doble acristalamiento.

Puede ser templado y laminado.

Su transmisión luminosa es superior a los obtenidos por proceso pirolítico.- Reducen drásticamente las pérdidas del calor por causa de la radiación, siendo su coef. $\mathrm{K}$ inferior al de los pirolíticos para igualdad de características en la luna soporte.

Su utilización principal es para edificios con grandes fachadas acristaladas como muros cortina, o bien con grandes superficies acristaladas en las que las pérdidas térmicas deban reducirse al máximo.

Lafigura 14 muestra comparativamente las características óptico-energéticas globales entre un doble acristalamiento y dos dobles acristalamientos con capas de baja emisividad comercializados por una casa en España.

Estas afirmaciones son ciertas solamente en invierno ya que, por el funcionamiento de estos vidrios (con la capa de baja emisividad colocada en la cara exterior de la luna interior del doble acristalamiento), no permiten la reemisión al exterior de la energía absorbida en la luna, emisión que como se dijo en la primera parte está en función del 


\begin{tabular}{|c|c|c|c|c|c|c|c|c|c|c|}
\hline $\begin{array}{c}\text { Tpo de } \\
\text { acristalamionto }\end{array}$ & Composición & $\underset{c}{\mathrm{kcal} / \mathrm{hm} \mathrm{m}^{2}}$ & $\underset{x}{\mathbf{F a}}$ & $\begin{array}{l}\mathbf{n} \\
\mathbf{x}\end{array}$ & $\begin{array}{l}\text { RE } \\
\%\end{array}$ & $\begin{array}{l}\text { AE } \\
\mathbf{X}\end{array}$ & $\begin{array}{l}\text { TE } \\
\mathbf{X}\end{array}$ & $\begin{array}{l}\text { Fs } \\
\times\end{array}$ & $\begin{array}{l}\text { Aislamiento } \\
\text { acítico } \\
\text { di }\end{array}$ & $\begin{array}{l}\text { Peso } \\
\mathrm{kg} / \mathrm{m}^{2}\end{array}$ \\
\hline \multirow{2}{*}{$\begin{array}{l}\text { CUmaut } \\
\text { tradicional } \\
\text { (doble } \\
\text { acristalamiento } \\
\text { ordinario) }\end{array}$} & $4-12-4$ & 2,6 & 15 & 82 & 13 & 15 & 72 & 76 & 32 & 21 \\
\hline & $6-12-6$ & 2,6 & 15 & 80 & 13 & 20 & 67 & 72 & 33 & 31 \\
\hline \multirow{2}{*}{$\begin{array}{l}\text { CUMAuT } \\
\text { PLUS con EKO } \\
\text { (capa de baja } \\
\text { emisividad } \\
\text { pirolitica) }\end{array}$} & $4-12-4$ & 1,9 & 20 & 74 & 14 & 20 & 66 & 74 & 32 & 21 \\
\hline & $6-12-6$ & 1,9 & 20 & 71 & 14 & 24 & 62 & 72 & 33 & 31 \\
\hline \multirow{2}{*}{$\begin{array}{l}\text { CLMALT PLUS } \\
\text { con PLANITHaRu } \\
\text { (capa de baja } \\
\text { omisividad } \\
\text { catódica) }\end{array}$} & $4-12-4$ & 1,5 & 11 & 79 & 21 & 27 & 52 & 66 & 32 & 21 \\
\hline & $6-12-6$ & 1,5 & 11 & 77 & 20 & 32 & 48 & 62 & 33 & 31 \\
\hline
\end{tabular}

Fig. 14.- Características óptico-energéticas de tres dobles acristalamientos de " Cristalería Española ".

sobrecalentamiento del vidrio respecto del medio por la absorción de parte de la energía solar incidente, por ello mientras los vidrios de baja emisividad sean comercializados en dobles acristalamientos habrá que cuidar las protecciones solares del hueco (voladizos, toldos, etc) de forma que no incida la radiación solar directa sobre el hueco en verano, evitando la absorción de parte del flujo incidente y el sobrecalentamiento del local por la parte de la radiación que ha sido transmitida al interior a través del vidrio.

Si se comercializaran como vidrios simples, lo cual podría hacerse con los obtenidos por procedimiento pirolítico, este problema se podría obviar con un mecanismo de reversibilidad para las lunas incluido en la carpintería, con lo que en invierno la capa de baja emisividad podría colocarse al exterior y en verano al interior sin ningún problema, dejando de ser un condicionante para las fachadas de los edificios las protecciones solares.

\section{PARTE III.- EL FUNCIONAMIENTO TÉRMICO DE LOS ACRISTALAMIENTOS (VENTANAS)}

La superficie acristalada es uno de los componentes de la fachada que cumple numerosas funciones, y su comportamiento térmico está condicionado por el acristalamiento que tenga.

Las funciones principales de los acristalamientos son:

-Iluminación natural de los locales

-Visibilidad sobre el mundo exterior

La ventana es la principal responsable de las pérdidas calorificas de los edificios.

La pared acristalada ha sido uno de los componentes del edificio cuya evolución ha sido más rápida. El simple acristalamiento está dando paso al doble acristalamiento.

Hoy es corriente hablar de cristales de baja emisividad en Europa, no tanto en España, y para mañana se habla de cristales más perfectos: "cristales a vida" de transparencia variable.

El problema puesto a los fabricantes y usuarios de acristalamientos es el conocimiento de sus características, y más concretamente de las características térmicas, que como ya se ha dicho son evaluadas por:

-El coeficiente de transmisión de calor $(\mathrm{K})$.

$-\mathrm{El}$ factor solar (S).

-La transmisión luminosa. 


\section{COEFICIENTE DE TRANSMISIÓN TÉRMICA} (K).

Las pérdidas de una pared acristalada se expresan por el coef. $K$ (coef. de transmisión por unidad de superficie $y$ grado centígrado de diferencia de temperatura entre las caras del elemento superficial). Este puede ser:

-El coef. $\mathrm{K}$ de la ventana sola (ventanas en exterior, alojamientos, etc.).

-El coef. K medio día-noche (teniendo en cuenta visillos, cortinas y cerramientos eventuales). Es por ejemplo, el caso de acristalamientos de viviendas.

\section{1.- Coeficiente $K$ de la ventana sola.}

El coef. de las paredes acristaladas se refiere a la superficie del hueco; ello resulta de las pérdidas por transmisión a través de los elementos de la ventana:

-del vidrio, caracterizado por el coef. $\mathrm{K}_{\mathrm{v}}$ -de la carpintería, caracterizado por el coef. $K_{\mathrm{m}}$

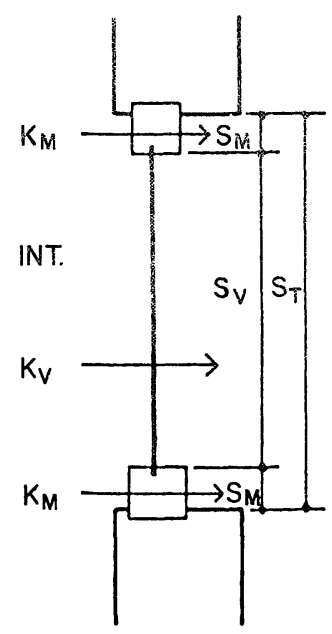

Fig.15.- El coeficiente $\mathrm{K}$ de una ventana.

El esquema de la figura 15, ilustra las dimensiones y superficies de las que se habla.

El coef. $K$ de la "ventana sola" $\left(\mathbf{K}_{\mathbf{n}}\right)$ se obtiene por la relación:

$\mathrm{K}_{\mathrm{n}}=\left(\mathrm{K}_{\mathrm{v}} \mathrm{S}_{\mathrm{v}}+\mathrm{K}_{\mathrm{m}} \mathrm{S}_{\mathrm{m}}\right) / \mathrm{S}_{\mathrm{T}}$

donde

$\mathrm{K}_{\mathrm{n}}=\mathrm{K}_{\mathrm{v}} \sigma+\mathrm{K}_{\mathrm{m}}(1-\sigma)$ con

$\sigma=\mathrm{S}_{\mathrm{v}} / \mathrm{S}_{\mathrm{T}}$

es decir, es la relación entre la superficie de ventana y la superficie de hueco.

\section{1.a.- Coef. $\mathrm{K}$ del acristalamiento $\left(\mathrm{K}_{\mathrm{v}}\right)$.}

Las pérdidas por el acristalamiento (caso del doble acristalamiento) resultan de los dos fenómenos ilustrados por el esquema de la figura 16.

-El coef. $\mathrm{K}_{\mathrm{c}}$ representa las pérdidas superficiales en partidas corrientes a través de los vidrios.

-El coef. $\mathrm{k}$ representa las pérdidas lineales debidas al puente térmico producido por el separador.

El valor del coef. $K_{v}$ viene dado por la expresión:

$$
K_{v}=K_{c}+W_{2} P / A
$$

donde $\mathrm{P}$ A son respectivamente el perímetro y superficie del hueco.

Examinemos sucesivamente la influencia de los dos componentes del coef. $\mathrm{K}_{\mathrm{v}}$ :

*Coeficiente lineal $(\mathrm{k})$ :

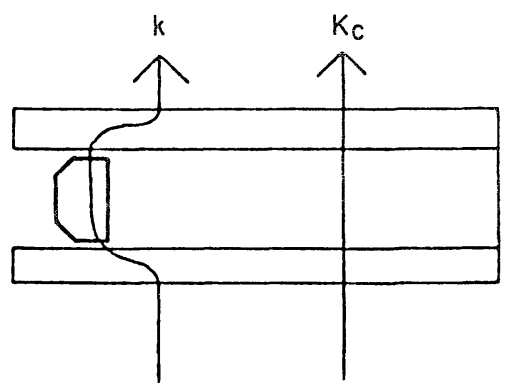

Fig.16.- El coeficiente $\mathrm{K}$ de un doble acristalamiento.

Con las tecnologías actuales se emplean dos tipos de intercaladores:

intercalador de masilla intercalador metálico

El valor del coef. $k$ varía del valor 0,02 a $0,05 \mathrm{~W} / \mathrm{m}^{\circ} \mathrm{C}$ sobre una influencia de $\mathrm{K}_{\mathrm{v}}$ entre $0,1 \div 0,25 \mathrm{~W} / \mathrm{m}^{2}{ }^{\circ} \mathrm{C}$ para una relación media P/A de 5,0.

*Coeficiente superficial en partida corriente $\left(\mathrm{K}_{\mathrm{c}}\right)$ : 
El valor del coef. $K_{c}$ está dado por la expresión:

$1 / K_{c}=1 / h_{i}+\Sigma r_{v}+r_{1}+1 / h_{c}$

$.1 / h_{1}$ y $1 / h_{e}$ representan los cambios superficiales (interior y exterior); estos valores son convencionales. Apenas se pueden mejorar (sobre todo en doble acristalamiento).

.$\Sigma \mathbf{r}_{v}$ representa la suma de las resistencias de los cristales. Aumentar este término supone un aumento de los espesores.

.Queda el término $r_{1}$ que representa la resistencia térmica de la cámara de aire (o gas).

Esta resistencia térmica, o mejor su inverso, es decir, la conductancia es la resultante de fenómenos complejos que pueden ser resumidos en dos términos:

La conducción/convección en el gas. Es función de características físicas de éste:

\section{-conductividad \\ -calor específico \\ -densidad \\ -viscosidad}

La radiación entre caras internas de los cristales. Este fenómeno depende en particular de la emisividad de las superficies (e). a) Influencia del gas.

Una de las soluciones consiste en sustituir el aire por un gas que tenga características que permita disminuir los intercambios por conducción/convección. El más extendido es el Argón.

Como ejemplo se dael coef. $K_{c}$ de un doble acristalamiento 4-12-4 $\mathrm{mm}$ para diferentes gases:

$$
\begin{array}{ll}
\text { - Aire } & \mathrm{K}_{\mathrm{c}}=3,05 \mathrm{~W} / \mathrm{m}^{2}{ }^{\circ} \mathrm{C} \\
\text { - Argón } & \mathrm{K}_{\mathrm{c}}=2,8 \mathrm{~W} / \mathrm{m}^{2}{ }^{\circ} \mathrm{C} \\
\text { - S F } & \mathrm{K}_{\mathrm{c}}=2,7 \mathrm{~W} / \mathrm{m}^{2}{ }^{\circ} \mathrm{C}
\end{array}
$$

\section{b) Influencia de la emisividad:}

El hecho de utilizar en un doble acristalamiento uno de los cristales, cuya superficie interna tiene una débil emisividad, permite reducir más o menos los intercambios por radiación.

Las emisividades corrientes actuales de cristales con capa bajo emisiva están comprendidas entre $\mathbf{e}=0,1$ y e $\mathbf{0} \mathbf{0}, 35$.

A título de ejemplo damos el coef. $K_{c}$ para diferentes combinaciones de acristalamientos 4-12-4 con cámara de aire:

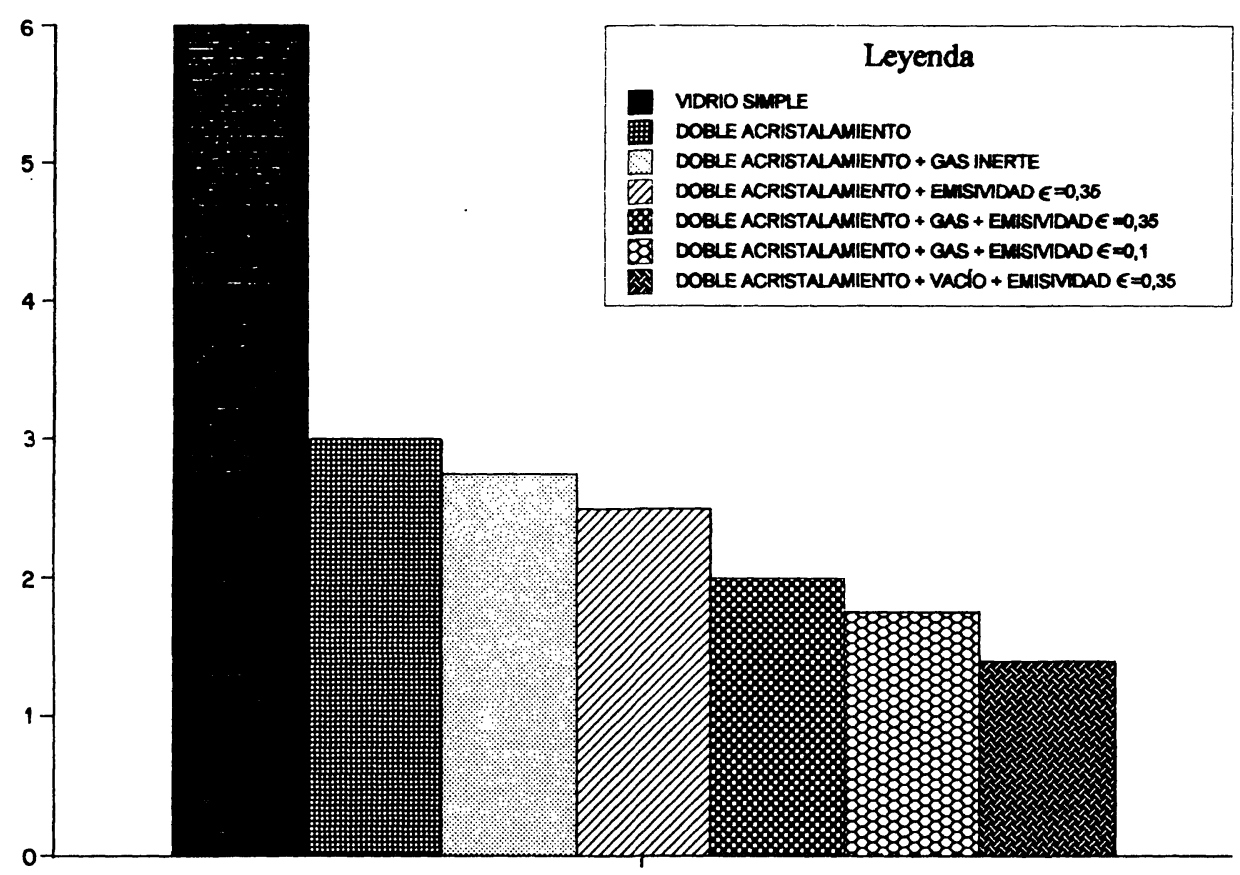

Fig.17.- Evolución del coeficiente $\mathrm{K}_{\mathrm{c}}$ en función de diferentes combinaciones de vidrios. 


\section{-2 cristales claros}

$$
\mathrm{K}_{\mathrm{c}}=3,05 \mathrm{~W} / \mathrm{m}^{2}{ }^{\circ} \mathrm{C}
$$

-1 vidrio claro +

+1 vidrio $\operatorname{con} \varepsilon=0,35$

$\mathrm{K}_{\mathrm{c}}=2,30 \mathrm{~W} / \mathrm{m}^{2}{ }^{\circ} \mathrm{C}$

-1 vidrio claro +

+1 vidrio con $\varepsilon=0,1 \quad \mathrm{~K}_{\mathrm{c}}=1,85 \mathrm{~W} / \mathrm{m}^{2}{ }^{\circ} \mathrm{C}$

La figura 17 muestra la evolución del coef. $\mathrm{K}_{\mathrm{c}}$ en función de diferentes combinaciones de acristalamientos:

\section{1.-Vidrio simple}

2.-Doble acristalamiento claro

3.-Doble acristalamiento + gas inerte

4.-Doble acristalamiento + emisividad $\varepsilon=0,35$

5.-Doble acristalamiento + gas + emisividad $\varepsilon=0,35$

6.-Doble acristalamiento + gas + emisividad $\varepsilon=0,1$

7.-Doble acristalamiento + gas + emisividad $\varepsilon=0,35$

\section{1.b.- Coeficiente $K$ de las carpinterías.}

Las pérdidas por la carpintería y por degradación (en PVC) se pueden expresar:

$$
\mathrm{d}_{\mathrm{m}}=\mathrm{k}_{\mathrm{m}} \mathrm{A}_{\mathrm{m}}
$$

En los elementos constitutivos de la carpintería(traviesas, montantes) siendo sensiblemente diferentes unos de otros, tanto sobre el plano geométrico como en su constitución, es difícil de calcular directamente el coef. de transmisión superficial $\mathrm{K}_{\mathrm{m}}$; así que preferimos la noción de coef. lineal de elementos “ $d_{m}$ ", el cual se escribe:

$$
\mathrm{d}_{\mathrm{m}}=\mathrm{H} \Sigma \mathrm{k}_{\mathrm{v}}+\left(\mathrm{L}-\Sigma \mathrm{l}_{\mathrm{app}}\right) \Sigma \mathrm{k}_{\mathrm{h}}
$$

.con $\mathbf{H}$ y $\mathbf{L}$ respectivamente la altura y la longitud del hueco de ventana

.$\Sigma \mathrm{l}_{\text {app }}$ es la suma de las longitudes de los elementos verticales

. $\mathbf{k}_{\mathbf{v}} \mathbf{y} \mathbf{k}_{\mathbf{h}}$ son los coefícientes $\mathrm{k}$ de elementos verticales $\mathrm{y}$ horizontales.

A partir de la siguiente relación podemos obtener el coef. $\mathbf{K}_{\mathbf{m}}$ medio para la ventana por:

$$
K_{m}=\left[H \Sigma k_{v}+\left(L-\Sigma l_{\text {app }}\right) \Sigma k_{h}\right] / A_{m}
$$

De forma general la expresión de $\mathrm{k}_{\mathrm{v}} \circ \mathrm{k}_{\mathrm{h}}$ es la siguiente:

$$
1 / k=1 / h_{\mathbf{i}} \mathbf{I}_{\mathbf{i}}+\mathbf{1} / \Sigma \mathbf{C}+\mathbf{1} / \mathbf{h}_{\mathrm{e}} \mathbf{l}_{\mathbf{e}}
$$

.$_{\mathbf{i}}$ ' y $\mathbf{h}_{\mathbf{e}}$ ' son los coeficientes de intercambio superficial

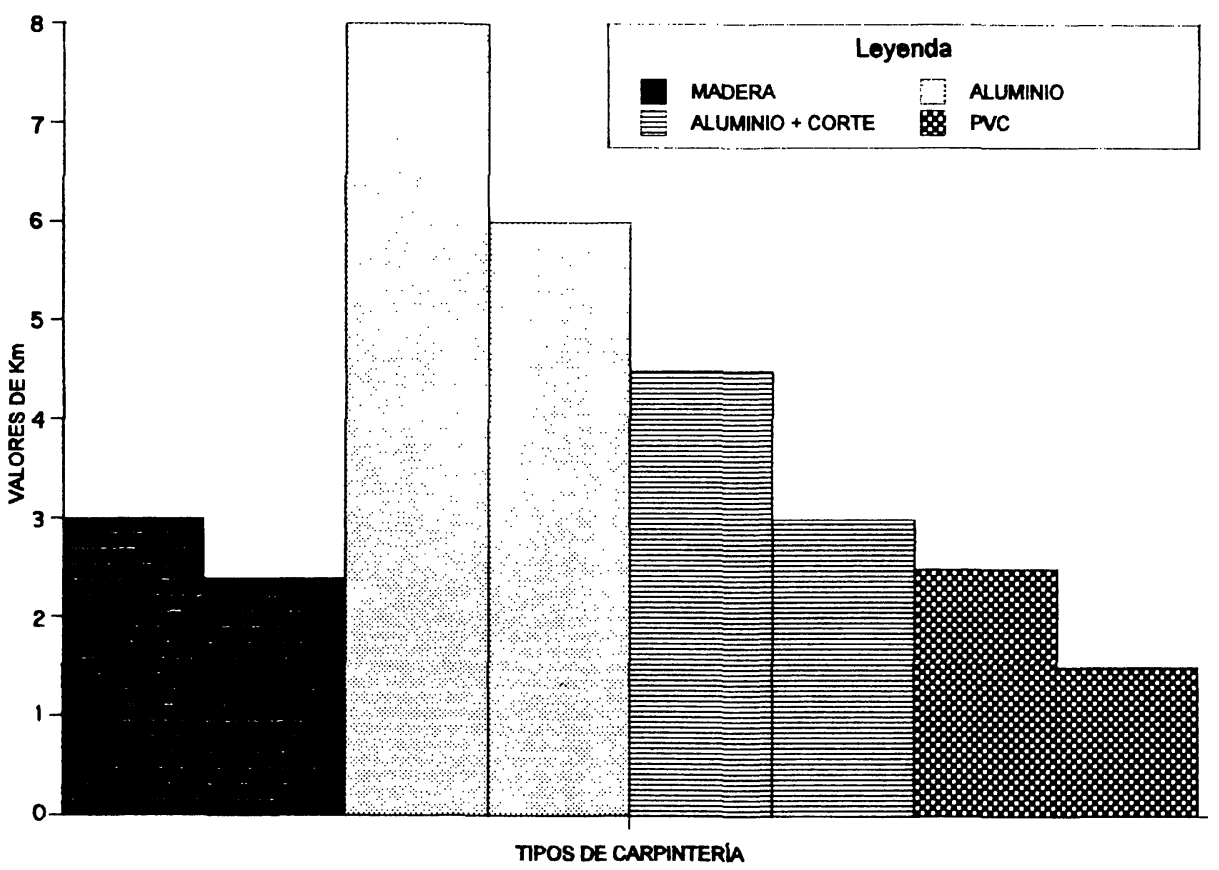

Fig. 18.- Límites del valor del coeficiente $\mathrm{K}_{\mathrm{m}}$ para cuatro tipos de carpinterías ordinarias. 
interior y exterior respectivamente.

$I_{i}$ y $I_{e}$ los desarrollos interior y exterior.

$\Sigma \mathbf{C}$ suma de las conductancias.

Siguiendo la naturaleza de las carpinterías (madera, metálica, metálica con rotura del puente térmico, PVC) cada uno de los tres términos tendrá una importancia preponderante sobre la calidad térmica de la carpintería:

\section{*Carpintería de madera:}

$\Sigma \mathbf{C}$ depende principalmente del grosor de la carpintería.

\section{*Carpintería metálica:}

$1 / \Sigma \mathrm{C}$ es próximo a cero, de ahí la importancia de los desarrollos interior y exterior (efecto de alas de radiador).

*Carpintería metálica con rotura de puente térmico:
$\Sigma \mathbf{C}$ será más débil cada vez que los cortes sean delgados y largos y el material constitutivo sea aislante.

*Carpintería de PVC:

$\Sigma$ Ces función del número de cámaras dentro del grosor de la carpintería. La presencia de refuerzos metálicos degrada el coef. $K_{m}$

El gráfico de la figura 18 da para los cuatro tipos de carpintería los rangos de variación del coeficiente medio $\mathrm{K}_{\mathrm{m}}$.

\section{1.c.- Coeficiente $\mathrm{K}$ de las "ventanas solas".}

Habida cuenta de los coeficientes $\mathrm{K}_{\mathrm{v}}$ y $\mathrm{K}_{\mathrm{m}}$ definidos anteriormente, la siguiente tabla muestra los valores del coef. $\mathrm{K}$ de la "ventana sola" (ventana media de 1,4 x 1,4 $\mathrm{m}^{2}$ ) equipada con doble acristalamiento 4-12-4:

\begin{tabular}{|l|c|c|c|}
\hline $\begin{array}{c}\text { Naturaleza de la } \\
\text { carpintería }\end{array}$ & $\begin{array}{c}\text { Doble } \\
\text { acristalamiento claro }\end{array}$ & $\begin{array}{c}\text { Doble acristalamiento donde } \\
\text { un cristal tiene } \\
\epsilon=0,35\end{array}$ & $\begin{array}{c}\text { Doble acristalamiento } \\
\text { donde un cristal tiene } \\
\epsilon=0,1\end{array}$ \\
\hline Madera & 2,95 & 2,45 & 2,15 \\
Metálica & 4,30 & 3,85 & 3,55 \\
M.c.r.p.t. & 3,30 & 2,80 & 2,50 \\
P.V.C. & 2,80 & 2,35 & 2,05 \\
\hline
\end{tabular}

\section{2.- Coeficiente $K$ medio dia/noche.}

Se define el coef. medio día/noche, mediante la media ponderada del coef. $\mathrm{K}$ medio de día y del coef. $\mathrm{K}$ medio de noche.

$\mathrm{El}$ coeficiente $\mathrm{K}$ de día está condicionado por la presencia de visillos sobre una parte de la ventana. El cálculo del coef. $\mathrm{K}$ de día $\left(\mathrm{K}_{\text {da }}\right)$ se obtiene añadiendo a la resistencia media de la "ventana sola" un valor fijo de 0,025 :

$$
1 / \mathrm{K}_{\text {dia }}=1 / \mathrm{K}_{\mathrm{h}}+0,025
$$

El coef. $K$ de noche $\left(K_{\text {noche }}\right)$ tiene, o no, en cuenta la presencia de cierres. Si no los hay, los cálculos se hacen suponiendo la presencia de cortinas pesadas sobre cierta parte de la ventana. Un valor fijo es añadido a la resistencia media de la "ventana sola":

$$
1 / \mathrm{K}_{\text {noche }}=1 / \mathrm{K}_{\mathrm{h}}+0,05
$$

Con cierres exteriores (puerta de ventana exterior o persianas enrollables de láminas que se juntan), el coef. $\mathrm{K}_{\text {noche }}$ tiene en cuenta: el cierre, la cámara de aire creada entre la ventana y el cierre, el visillo y la cortina interior; su expresión es la siguiente:

$$
\mathrm{K}_{\text {noche }}=\mathrm{K}_{\mathrm{o}}+\mathrm{JK}_{\mathrm{o}}{ }^{2} / \mathrm{K}_{\mathrm{e}}
$$

J tiene en cuenta la ventilación de la cámara de aire, es decir, la parte de permeabilidad al aire del cierre exterior, de ahí los tres casos prescritos en las reglas de cálculo: 
-Cierres fuertemente permeables

-Cierres de permeabilidad media

-Cierres de muy baja permeabilidad

El coeficiente medio día/noche es la media aritmética de $\mathrm{K}_{\text {dia }} \mathrm{y} \mathrm{K}_{\text {noche }}$ :

$$
K_{\text {dia/noche }}=1 / 2\left(K_{\text {dia }}+K_{\text {noche }}\right)
$$

\section{FACTOR SOLAR (S).}

A fin de tener en cuenta los aportes energéticos en la evaluación de las necesidades, es necesario conocer el factor solar S de la ventana.

El factor solar se define como la relación entre la energía entrante en un local a través de la superficie que se considera, (el soleamiento durante el período de calentamiento) y de la energía solar incidente durante el mismo período.

\section{1.- Cálculo del factor solar.}

El cálculo consiste en determinar sucesivamente:

-El factor solar del acristalamiento

-El factor solar de la superficie acristalada sola -El factor solar de la superficie acristalada colocada in situ.

\section{1.a.- Factor solar del acristalamiento.}

La medida obtiene el factor solar "normal" del acristalamiento $S_{n v}$ que corresponde a una incidencia normal de la radiación solar.

A partir de éste se determina el factor solar medio del acristalamiento $S_{v}$ correspondiente noya a una incidencia normal de radiación solar, sino al conjunto de la incidencias en el período de calentamiento.

Por falta de haber establecido un método de cálculo preciso, se admite que:

-en acristalamiento simple $\mathrm{S}_{\mathrm{v}}=0,90 \mathrm{~S}_{\mathrm{nv}}$ -en acristalamiento doble $S_{v}=0,85 S_{n v}$

\section{1.b.- Factor solar de la superficie acristalada sola.}

Se trata del factor solar "medio" en su sentido más amplio, de la superficie acristalada con su carpintería, pero sin visillos ni encuadramiento (de jambas, dintel y alféizar).
Esta es su expresión:

$$
S_{n}=S_{v} \sigma+S_{m}(1-\sigma)
$$

en la cual:

* $\mathrm{S}_{\mathrm{v}}$ es el factor solar medio del acristalamiento * $\mathrm{S}_{\mathrm{m}}$ es el factor solar medio de la carpintería

* $\sigma$ es la relación de la superficie de claridad y la superficie del hueco.

El factor solar de la carpintería se toma de los valores siguientes:

-Carpintería de madera $0,08 \mathrm{~K}_{\mathrm{m}}$

-Carpintería metálica sin corte de puente térmico $0,20 \mathrm{~K}_{\mathrm{m}}$ -Carpintería metálica con ruptura de puente térmico o en plástico $0,03 \mathrm{~K}_{\mathrm{m}}$, siendo $\mathrm{K}_{\mathrm{m}}$ el coef. $\mathrm{K}$ de la carpintería. Para las carpinterías con ruptura de puentes térmicos $\mathrm{K}_{\mathrm{m}}$ secalcula siguiendoel métododesarrollado anteriormente.

2.1.c.- Factor solar de la superficie acristalada colocada in situ.

Este factor solar S, es igual al factor solar de la superficie acristalada sola $S_{n}$, eventualmente corregido para tener en cuenta los visillos y el encuadramiento.

-Incidencia de los visillos.

Esta incidencia solo se tiene en cuenta en las estancias habitables de los alojamientos y se traduce por una reducción del $5 \%$.

-Incidencia del encuadramiento.

Se admite que esta incidencia se traduce por una reducción del factor solar:

-Igual al $10 \%$ para las superficies acristaladas colocadas a haces interiores del cerramiento.

-Nula para aquellas puestas a haces exteriores del cerramiento.

Las superficies acristaladas puestas en situación intermedia y las dobles ventanas con marcos separados son consideradas situadas al interior y aquellas situadas en fachadas de poco espesor son consideradas colocadas en el paramento exterior.

- Expresión de $S$.

Teniendo en cuenta lo comentado éstas son las diversas expresiones de $\mathrm{S}$ : 


\begin{tabular}{|l|l|l|}
\hline & \multicolumn{1}{|c|}{ Superficie Acristalada } & \multicolumn{1}{c|}{ Expresión de S } \\
\hline \multirow{3}{*}{ Estancia habitables } & Interior & $\mathrm{S}=0,855 \mathrm{~S}_{\mathrm{n}}$ \\
\cline { 2 - 3 } & Exterior & $\mathrm{S}=0,95 \mathrm{~S}_{\mathrm{n}}$ \\
\hline \multirow{3}{*}{ Otros locales } & Interior & $\mathrm{S}=0,9 \mathrm{~S}_{\mathrm{n}}$ \\
\cline { 2 - 3 } & Exterior & $\mathrm{S}=\mathrm{S}_{\mathrm{n}}$ \\
\hline
\end{tabular}

\section{2.- Valores corrientes del factor solar.}

Los valores de S que figuran en el capitulo VII del DTU Reglas ThK 77 correspondientes a superficies acristaladas corrientes, en particular en lo que concierne a los valores de la relación entre claridad (superficie efectiva acristalada) y la superficie del hueco: , los valores $S_{n v}$ son iguales $a$ :

- Acristalamiento simple: 0,90
- Acristalamiento doble: 0,81

\section{TRANSMISIÓN LUMINOSA.}

La transmisión luminosa de las ventanas (en general de las superficies acristaladas) depende exclusivamente de los vidrios utilizados. Las características ópticas son las expresadas en la parte I a efectos de transmisión luminosa

\section{BIBLIOGRAFIA}

(1) "Parametres caracteristiques et fonctionnement des vitrages." Seminaire spécialisé Nouvelles performances thermique des vitrages, fenêtres et fermetures: JOURNEES “ISOLATION" 88. J. L. CHEVALIER

(2) "Les vitrages faiblement émissifs par dépôt pyrolytique en continu." JOURNEES "ISOLATION" 88. J. FREMAUX

(3) "Le fonctionnement thermique des fenêtres". CSTB. G. Tramblay.

(4) "Règle de calcul des caractéristiques thermiques utiles des parois de construction". Libreta del CSTB n² 2096 de septiembre de 1986.

(5) "Détermination du facteur de transmission lumineuse et du facteur solaire conventionnel des vitrages". Proyecto de norma francesa de Junio de 1987.

(6) "Manual del vidrio". Editado por el Centro de Información Técnica de Aplicaciones del Vidrio (CITAV).

(7) "Influence of Windows on Buildings Energy Use". S. Selkowitz. Lawrence Berkeley Laboratory (University of California)

(8) "Windows for Energy Efficient Buildings" Vol 1. $\mathrm{N}^{\circ} 1$

(9) "Les vitrages faiblement emissifs par depot pyrolytique en continu". J. Fremaux. Agence francaise pour la maitrise de l'energie.

(10) "Soluciones del vidrio". Cristaleria Española.

(11) "EKO, PLANITHERM. La alternativa de la baja emisividad". Cristalería Española.

(12) "Construir con luz". ARIPLAK. 